\title{
Precessing cylinders at the second and third resonance: Turbulence controlled by geostrophic flow
}

\author{
Jianfei Jiang, ${ }^{1}$ Dali Kong, ${ }^{2}$ Rixiang Zhu, ${ }^{3}$ and Keke Zhang ${ }^{4, *}$ \\ ${ }^{1}$ Institute of Geology and Geophysics and University of Chinese Academy of Sciences, Beijing, China \\ ${ }^{2}$ Center for Geophysical and Astrophysical Fluid Dynamics, University of Exeter, Exeter, United Kingdom \\ ${ }^{3}$ Institute of Geology and Geophysics, Chinese Academy of Sciences, Beijing, China \\ ${ }^{4}$ Center for Geophysical and Astrophysical Fluid Dynamics, University of Exeter, Exeter, United Kingdom, \\ and Lunar and Planetary Science Laboratory, Macau University of Science and Technology, Taipa, Macau \\ (Received 29 November 2014; revised manuscript received 30 March 2015; published 8 September 2015)
}

\begin{abstract}
We investigate, via both asymptotic analysis and direct numerical simulation, precessionally driven flow of a homogeneous fluid confined in fluid-filled circular cylinders that rotate rapidly about their symmetry axis and precess about a different axis and that are marked by radius-height aspect ratios $\Gamma=1.045945$ and $\Gamma=1.611089$. At these radius-height aspect ratios, the Poincaré force resonates directly with the two special inertial modes that have the simplest vertical structure. An asymptotic analytical solution in closed form describing weakly precessing flow is derived in the mantle frame of reference for asymptotically small Ekman numbers, showing quantitative agreement with the result of direct nonlinear numerical simulation. Our numerical simulation makes use of a finite-element method with the three-dimensional tetrahedralization of a cylindrical cavity that allows the construction of dense nodes in the vicinity of the bounding surface of the cavity for resolving the thin viscous boundary layer. It is found that axisymmetric geostrophic flow in the alternating eastward and westward direction can be generated and maintained by nonlinear and viscous effects in the viscous boundary layer. It is also found that, when the precessing rate is moderate and, consequently, the geostrophic flow is weak, nonlinear interaction between the resonant inertial mode and the nonesonant inertial modes driven by the Poincare force and the boundary-layer influx leads to strongly turbulent flow with irregular temporal-spatial fluctuation. When the cylinders are strongly precessing such that the geostrophic flow becomes predominant, however, the effect of the geostrophic flow controls/stabilizes its nonlinear dynamics, leading to weakly turbulent flow that can be largely described by a dominant quasisteady geostrophic component and a weak nonaxisymmetric component localized in the region where the geostrophic flow is weak.
\end{abstract}

DOI: 10.1103/PhysRevE.92.033007

PACS number(s): 47.32.Ef

\section{INTRODUCTION}

The problem of precessionally driven flow in fluid-filled circular cylinders has several important applications and has been studied for many decades (for example, [1-9]. Since both the thermal and the electrical conductivities in the Earth's liquid core are two to three times higher than previous estimates, which severely restricts the thermal power available for sustaining the geodynamo [10], geomagnetic fields may be generated by lunar-solar precession-driven flow (see, for example, [11]). It is also believed that the lunar dynamo may once have been driven by the Earth-driven precession in its liquid core [12]. Nore et al. [13], using a spectral and finite-element hybrid method, demonstrated that dynamo action is possible in a precessing cylinder (see also [14] and [15]). The precession-driven flow in rotating cylinders is also of industrial interest as it is closely related to the stability properties of spinning spacecraft with fluid payloads. Since the effect of Coriolis forces is dynamically predominant in many rotating planets, the problem also provides an instructive paradigm for understanding the general property of Coriolis-force-controlled turbulence in rapidly rotating systems. Moreover, because the rotation axis of a fluid container in laboratory experiments is usually not parallel to the rotation axis of the Earth, the precessing flow always

\footnotetext{
*kzhang@ex.ac.uk
}

takes place in the experimental study of rotating flow on the Earth $[16,17]$.

The present study considers a homogeneous fluid of viscosity $v$ confined in a fluid-filled circular cylinder of height $d$ and radius $\Gamma d$ that rotates rapidly at angular velocity $\boldsymbol{\Omega}_{0}$ about its symmetry axis and precesses at angular velocity $\boldsymbol{\Omega}_{p}$ about a different axis that is fixed in space. There exist three key dimensionless parameters that characterize the problem: the Ekman number $E=v /\left|\boldsymbol{\Omega}_{0}\right| d^{2}$, providing the measure of relative importance between the typical viscous force and the Coriolis force; the Poincaré number Po $=\left|\boldsymbol{\Omega}_{p}\right| /\left|\boldsymbol{\Omega}_{0}\right|$, quantifying the strength of the precessional forcing; and the geometrical parameter $\Gamma$, representing the radius-height aspect ratio of the cylinder. As long as the Ekman number $E$ is sufficiently small such that its dynamics is dominated by rotational effects, the precise value of $E$ becomes physically and mathematically less significant. This is because the primary properties of the precessing flow can be approximately described by the relevant asymptotic behavior for $0<E \ll 1$ [7]. Most laboratory experiments were performed in the range $10^{-5} \leqslant E \leqslant 10^{-4}$ (for example, [4,5,9]). Similar to the Rayleigh number in the problem of thermal convection, the size of the Poincaré number Po controls the degree of nonlinearity of precessing flow, which has been adopted as the key parameter in the many existing experimental and numerical studies. By contrast, the geometrical parameter $\Gamma$, an important parameter in controlling the resonance and the nonlinear behavior of precessing flow, has not received 
sufficient attention. For example, perhaps for the reason of experimental convenience, Manasseh [3] used a cylinder with aspect ratio $\Gamma=0.375$; Kobine [4], with $\Gamma=0.385$; and Mouhali [8], with $\Gamma=0.427$.

It proved instructive to characterize the spatial structure of a cylindrical inertial mode using the triple index notation $\boldsymbol{u}_{m n k}$ [18-20], where $\boldsymbol{u}_{m n k}$ denotes the velocity of the inertial mode. In this notation, the first index $m$ denotes the azimuthal wave number of the mode; $n$, its axial wave number; and $k$, its radial wave number. Two conditions must be satisfied in order that the Poincaré force resulting from precession can directly resonate with an inertial wave mode $\mathbf{u}_{m n k}$. The first condition is that the inertial mode $\mathbf{u}_{m n k}$ must have the azimuthal wave number $m=1$ with an odd axial wave number $n$. The second condition is that the frequency $\omega_{m n k}$ of the inertial mode $\mathbf{u}_{m n k}$ must be equal to 1 . With these two conditions, we can determine the resonant values of the aspect ratio,

$$
\Gamma=\frac{\xi_{1 n k}}{\sqrt{3} n \pi},
$$

where $n=1,3,5, \ldots$ and $\xi_{1 n k}$ is a solution of the equation

$$
\xi_{1 n k} J_{0}\left(\xi_{1 n k}\right)+J_{1}\left(\xi_{1 n k}\right)=0,
$$

with $J_{n}$ denoting the standard Bessel function and $k$ being labeled in ascending order,

$$
0<\xi_{1 n 1}<\xi_{1 n 2}<\xi_{1 n 3} \cdots<\xi_{1 n k} \ldots
$$

When $n=1$ and $k=1$, we have the smallest root $\xi_{111}=$ 2.73462 , which gives rise to $\Gamma=0.502559$. In other words, the fundamental resonance takes place in a cylinder of approximately equal diameter and height with radius-height aspect ratio $\Gamma=0.502559$, in which the precessional force resonates directly with the inertial mode $\boldsymbol{u}_{111}$. At the second primary resonance with $n=1$ and $k=2$ occurring in a cylinder with $\Gamma=1.045945$, the precessional force resonates directly with the inertial mode $\boldsymbol{u}_{112}$, while, at the third primary resonance with $n=1$ and $k=3$ in a cylinder with $\Gamma=1.611089$, the precessional force resonates directly with the mode $\boldsymbol{u}_{113}$. To simplify the notation, we denote $\Gamma_{1}=0.502559, \Gamma_{2}=$ 1.045945 , and $\Gamma_{3}=1.611089$. The problem of nonlinear precessing flow at the second and third resonance has not been investigated either experimentally or numerically. It is worth noting that the higher-order-mode resonances such as $\mathbf{u}_{114}$ and $\mathbf{u}_{115}$ with larger resonant $\Gamma$ along with larger $n$ and $k$ are much weaker [7] and, hence, less interesting.

It is important to point out that the resonant aspect ratios such as $\Gamma=\Gamma_{2}$ are determined in the limits Po $\rightarrow 0$ and $E \rightarrow 0$. In the general asymptotic solution for $0<E \ll 1$ describing the weakly precessing flow that satisfies the no-slip boundary condition and that is valid both near and far from resonance [7], it was revealed that there exist, according to the size of $\Gamma$, two characteristically distinct regimes. For values of $\Gamma>0$ and not within $O(\sqrt{E})$ of resonance, for example, $\left[\Gamma_{1}+O(\sqrt{E})\right]<\Gamma<\left[\Gamma_{2}-O(\sqrt{E})\right]$, the amplitude $|\boldsymbol{u}|$ of the weakly precessing flow is marked by the asymptotic scaling $|\boldsymbol{u}|=O$ (Po) for $0<E \ll 1$. In a way, the aspect ratio $\Gamma$ in the problem of a precessing cylinder plays a role similar to that of the eccentricity in the problem of a precessing spheroid [21]. When the circular cylinders are sufficiently close to the aspect ratio $\Gamma_{1}$ or $\Gamma_{2}$ or $\Gamma_{3}$ and when the Ekman number $E$ is also sufficiently small, the precessional force resonates directly with the cylindrical inertial modes with the simplest vertical structure, and hence, the amplitude of the precessing flow $|\boldsymbol{u}|$ obeys the asymptotic scaling $|\boldsymbol{u}|=O(\mathrm{Po} / \sqrt{E})$ for $0<$ $E \ll 1$. Since accurate resonant value like $\Gamma_{2}$ is determined in the limits $\mathrm{Po} \rightarrow 0$ and $E \rightarrow 0$, it is expected that there are no significant differences among the nonlinear solutions that are obtained at a finite $E$ and a sufficiently large Po within the parameter ranges $\left[\Gamma_{2}-O(\sqrt{E})\right]<\Gamma<\left[\Gamma_{2}+O(\sqrt{E})\right]$ and $\left[\Gamma_{3}-O(\sqrt{E})\right]<\Gamma<\left[\Gamma_{3}+O(\sqrt{E})\right]$, even though our actual computations are focused on the values $\Gamma=\Gamma_{2}$ and $\Gamma=\Gamma_{3}$. In other words, while the weakly precessing solutions in the analytical form are only valid at $\Gamma=\Gamma_{2}$ and $\Gamma=\Gamma_{3}$, the strongly precessing solutions obtained at $\Gamma=\Gamma_{2}$ and $\Gamma=\Gamma_{3}$ for sufficiently large values of Po would be robust, having properties similar to those obtained at slightly different aspects in the vicinities of $\Gamma_{2}$ and $\Gamma_{3}$.

An unusual nonlinear phenomenon was revealed in the precessing spherical-like cylinder with radius-height aspect ratio $\Gamma=\Gamma_{1}[22,23]$. For the purpose of providing clearer discussion and description, we introduce the following three definitions for this paper: (i) precessing flow that is dominated by a single inertial mode with a constant amplitude is referred to as laminar precessing flow; (ii) precessing flow that has a complicated spatial structure with nonperiodically varying amplitude, but a coherent and largely regular leading-order spatial structure, is referred to as weakly turbulent precessing flow; and (iii) precessing flow that is marked by irregular temporal-spatial fluctuation and can only be described in a statistical way, is referred to as strongly turbulent precessing flow. In the case of the aspect ratio $\Gamma_{1}$, it was found that interior rigid-body rotation-whose direction and magnitude are such that the angular momentum of the system is reducedalways dominates the nonlinear precessing flow. Despite an extensive and systematic search in a broad range of Poincaré numbers Po and Ekman numbers $E$, there is no indication of the existence of strongly turbulent precessing flow in the spherical-like cylinder with aspect ratio $\Gamma_{1}$ [23]. This raises several interesting questions. Are nonlinear behaviors in the second and third resonant cylinders with aspect ratios $\Gamma_{2}$ and $\Gamma_{3}$ significantly different? Can interior rigid-body rotation also exist in resonant cylinders with $\Gamma_{2}$ and $\Gamma_{3}$ ? Would the precessing flow become strongly turbulent in precessing cylinders at the second and third resonance?

The primary objective of the present study is to answer the above questions by investigating nonlinear precessing flow at the second resonance in a precessing cylinder with radiusheight aspect ratio $\Gamma_{2}$ and at the third resonance in a precessing cylinder with $\Gamma_{3}$. We reveal that the nonlinear precessing flow in cylinders with aspect ratio $\Gamma_{2}$ or $\Gamma_{3}$ differs characteristically from that in a spherical-like cylinder with $\Gamma_{1}$. In what follows we begin by presenting a mathematical formulation of the problem in Sec. II. An asymptotic solution in closed form describing the weakly precessing flow in a resonant cylinder with aspect ratio $\Gamma_{2}$ is presented in Sec. III. The nonlinear properties of the precessing flow in resonant cylinders with aspect ratios $\Gamma_{2}$ and $\Gamma_{3}$ are discussed in Sec. IV, and Sec. $V$ closes the paper with a brief summary and concluding remarks. 


\section{GOVERNING EQUATIONS IN THE MANTLE FRAME}

Consider a viscous, incompressible, and homogeneous fluid with density $\rho_{0}$ occupying a circular cylinder of length $d$ and radius $\Gamma d$ with radius-height aspect ratio $\Gamma$. We adopt cylindrical polar coordinates $(s, \phi, z)$ with the corresponding unit vectors $(\hat{\boldsymbol{s}}, \hat{\boldsymbol{\phi}}, \hat{z})$, in which $s=0$ is at the symmetry axis of the cylinders while $z=0$ is at the bottom surface. Our attention focuses on the two special cylinders with radius-height aspect ratios $\Gamma=\Gamma_{2}$ and $\Gamma=\Gamma_{3}$, representing the second and third primary resonance when $\Gamma$ gradually increases from a small value [7]. The first primary resonance takes place at $\Gamma=\Gamma_{1}$.

The cylinders rotate rapidly with the angular velocity $\boldsymbol{\Omega}_{0}=\Omega_{0} \hat{z}$, where $\Omega_{0}$ is constant about its axis of symmetry and undergoes precession slowly with an angular velocity $\boldsymbol{\Omega}_{p}$ that is fixed in an inertial frame at the angle $\alpha_{p}, 0<\alpha_{p} \leqslant \pi / 2$. Since the angle $\alpha_{p}$ does not play a key role in the dynamics of the problem, we concentrate on the case of $\alpha_{p}=\pi / 4$ throughout this paper. In the cylindrical polar coordinates fixed in the cylinder, which is usually referred to as the body or mantle frame of reference, the precession vector $\boldsymbol{\Omega}_{p}$ is time dependent,

$$
\begin{aligned}
\boldsymbol{\Omega}_{p}= & \left|\boldsymbol{\Omega}_{p}\right|\left[\hat{\boldsymbol{s}} \sin \alpha_{p} \cos \left(\phi+\Omega_{0} t\right)\right. \\
& \left.-\hat{\boldsymbol{\phi}} \sin \alpha_{p} \sin \left(\phi+\Omega_{0} t\right)+\hat{z} \cos \alpha_{p}\right],
\end{aligned}
$$

and the fluid motion driven by precession is governed by the two equations

$$
\begin{aligned}
\frac{\partial \boldsymbol{u}}{\partial t} & +\boldsymbol{u} \cdot \nabla \boldsymbol{u}+2\left(\hat{z} \Omega_{0}+\boldsymbol{\Omega}_{p}\right) \times \boldsymbol{u}+\frac{1}{\rho_{0}} \nabla p \\
& =v \nabla^{2} \boldsymbol{u}-2 \hat{\boldsymbol{z}}\left|\boldsymbol{\Omega}_{p}\right| \Omega_{0} s \sin \alpha_{p} \cos \left(\phi+\Omega_{0} t\right), \\
\nabla \cdot \boldsymbol{u} & =0,
\end{aligned}
$$

where $\boldsymbol{u}$ is the fluid velocity in the mantle frame and $p$ is the reduced pressure including the term $-s z \rho_{0}\left|\Omega_{p}\right| \Omega_{0} \sin \alpha_{p} \cos \left(\phi+\Omega_{0} t\right)$ resulting from the Poincaré forcing. The last term on the right-hand side of the equation of motion is known as the Poincaré forcing, which drives precessional flow against viscous dissipation. It is worth mentioning that, with the incompressible formulation chosen in this study, density $\rho_{0}$ plays no dynamic role.

There are two angular velocities $\boldsymbol{\Omega}_{0}$ and $\boldsymbol{\Omega}_{p}$ in the precession system, providing two possible time scales for scaling the governing equations. On employing the height $d$ as the length scale, $\Omega_{0}^{-1}$ as the unit of time, and $\rho_{0} d^{2} \Omega_{0}^{2}$ as the unit of pressure, we obtain the dimensionless equations:

$$
\begin{gathered}
\frac{\partial \boldsymbol{u}}{\partial t}+\boldsymbol{u} \cdot \nabla \boldsymbol{u}+2\left\{\hat{\boldsymbol{z}}+\operatorname{Po}\left[\hat{\boldsymbol{s}} \sin \alpha_{p} \cos (\phi+t)\right.\right. \\
\left.\left.-\hat{\boldsymbol{\phi}} \sin \alpha_{p} \sin (\phi+t)+\hat{z} \cos \alpha_{p}\right]\right\} \times \boldsymbol{u} \\
=-\nabla p+E \nabla^{2} \boldsymbol{u}-2 \hat{z} s \operatorname{Pos} \sin \alpha_{p} \cos (\phi+t), \\
\nabla \cdot \boldsymbol{u}=0 .
\end{gathered}
$$

In the body frame of reference, the flow on the bounding surface $\mathcal{S}$ of a precessing cylinder is at rest, imposing that

$$
\hat{z} \cdot \boldsymbol{u}=0 \quad \text { and } \quad \hat{z} \times \boldsymbol{u}=\mathbf{0}
$$

on the bottom at $z=0$ and the top at $z=1$ and

$$
\hat{\boldsymbol{s}} \cdot \boldsymbol{u}=0 \quad \text { and } \quad \hat{\boldsymbol{s}} \times \boldsymbol{u}=\mathbf{0}
$$

on the sidewall at $s=\Gamma$.

After deriving the weakly precessing solution at the second resonance with $\Gamma=\Gamma_{2}$ using an analytical method, we solve the problem defined by Eqs. (1) and (2) subject to the boundary conditions, Eqs. (3) and (4), via direct numerical simulation for $0<E \ll 1$ and $0<(\mathrm{Po} / \sqrt{E}) \leqslant O(10)$.

\section{AN ASYMPTOTICAL SOLUTION AT THE SECOND RESONANCE}

When Po is sufficiently small $(0<$ Po $\ll 1)$ with a low amplitude of the precessing flow $|\boldsymbol{u}| \ll 1$, the nonlinear equation, (1), may be linearized by omitting the higher order small terms, which results in the equations governing the weakly precessing flow,

$$
\begin{gathered}
\frac{\partial \boldsymbol{u}}{\partial t}+2 \hat{\boldsymbol{z}} \times \boldsymbol{u}+\nabla p=E \nabla^{2} \boldsymbol{u}-2 \hat{z} s \operatorname{Posin} \alpha_{p} e^{\mathrm{i}(\phi+t)}, \\
\nabla \cdot \boldsymbol{u}=0,
\end{gathered}
$$

where only the real part of the complex solution is taken as the physical solution of the problem, subject to the same no-slip boundary conditions, (3) and (4). Equations (5) and (6) are approximately valid when Po and $E$ are both sufficiently small, because of direct resonance together with the condition $0<$ $(\mathrm{Po} / \sqrt{E}) \ll 1$. An analytical solution in closed form for $(5)$ and (6), derived for the weakly resonant cylinder, would help us understand some aspects of the strongly precessing flow.

At the second resonance with $\Gamma=\Gamma_{2}$, the Poincaré force resonates directly with a single inertial mode $\left(p_{112}, \boldsymbol{u}_{112}\right)$, which is expressible as

$$
\begin{gathered}
p_{112}=J_{1}\left(\frac{\beta s}{\Gamma_{2}}\right) \cos (\pi z) e^{\mathrm{i} \phi} \\
\hat{\boldsymbol{s}} \cdot \boldsymbol{u}_{112}=-\frac{\mathrm{i}}{3}\left[\sqrt{3} \pi J_{0}\left(\frac{\beta s}{\Gamma_{2}}\right)+\frac{1}{s} J_{1}\left(\frac{\beta s}{\Gamma_{2}}\right)\right] \cos (\pi z) e^{\mathrm{i} \phi} \\
\hat{\boldsymbol{\phi}} \cdot \boldsymbol{u}_{112}=\frac{2}{3}\left[\sqrt{3} \pi J_{0}\left(\frac{\beta s}{\Gamma_{2}}\right)-\frac{1}{2 s} J_{1}\left(\frac{\beta s}{\Gamma_{2}}\right)\right] \cos (\pi z) e^{\mathrm{i} \phi} \\
\hat{\boldsymbol{z}} \cdot \boldsymbol{u}_{112}=-\mathrm{i} \pi J_{1}\left(\frac{\beta s}{\Gamma_{2}}\right) \sin (\pi z) e^{\mathrm{i} \phi}
\end{gathered}
$$

where $\mathrm{i}=\sqrt{-1}, \beta=5.69140$ represents the radial wave number of the mode, and $J_{n}$ denotes the standard Bessel function. In the asymptotic analysis, we assume that the resonant mode $\left(\boldsymbol{u}_{112}, p_{112}\right)$ is predominant and that nonresonant inertial modes are insignificant to the leading-order approximation. This assumption yields an asymptotic expansion for $0<E \ll 1$ in the form

$$
\begin{aligned}
& \boldsymbol{u}(s, \phi, z, t)=\left[\mathcal{A} \boldsymbol{u}_{112}(s, \phi, z)+\widehat{\boldsymbol{u}}+\widetilde{\boldsymbol{u}}\right] e^{\mathrm{i} t}, \\
& p(s, \phi, z, t)=\left[\mathcal{A} p_{112}(s, \phi, z)+\widehat{p}+\widetilde{p}\right] e^{\mathrm{i} t},
\end{aligned}
$$


where $\widehat{\boldsymbol{u}}$ and $\widehat{p}$ represent small interior perturbations, caused by viscous effects, to the resonant inertial mode $\left(\boldsymbol{u}_{112}, p_{112}\right)$, $\mathcal{A}$ is the amplitude of the precessing flow to be determined, and $\tilde{\boldsymbol{u}}$ and $\tilde{p}$ denote the flow and pressure, respectively, which are nonzero only within the viscous boundary layer. In the asymptotic expansion, the properties

$$
|\widehat{\boldsymbol{u}}| \ll\left|\mathcal{A} \boldsymbol{u}_{112}\right| \text { and }|\tilde{\boldsymbol{u}}|=\mathrm{O}\left|\mathcal{A} \boldsymbol{u}_{112}\right|
$$

are used in the mathematical analysis. Note that the inertial mode $\left(p_{112}, \boldsymbol{u}_{112}\right)$ given by (7)-(10) satisfies the equations

$$
\begin{aligned}
\mathrm{i} \boldsymbol{u}_{112}+2 \hat{z} \times \boldsymbol{u}_{112}+\nabla p_{112} & =0, \\
\nabla \cdot \boldsymbol{u}_{112} & =0
\end{aligned}
$$

in a rotating cylinder with $\Gamma=\Gamma_{2}$, as well as the boundary condition

$$
\hat{z} \cdot \boldsymbol{u}_{112}=0
$$

on the bottom at $z=0$ and the top at $z=1$ and

$$
\hat{\boldsymbol{s}} \cdot \boldsymbol{u}_{112}=0
$$

on the sidewall at $s=\Gamma_{2}$. Substitution of the asymptotic expansion into (5) and (6) yields the two sets of governing equations. The first set governs the small interior perturbation $\widehat{\boldsymbol{u}}$ and $\widehat{p}$ given by

$$
\begin{gathered}
\mathrm{i} \widehat{\boldsymbol{u}}+2 \hat{z} \times \widehat{\boldsymbol{u}}+\nabla \hat{p}=E \nabla^{2}\left(\mathcal{A} \boldsymbol{u}_{112}\right)-2 \hat{z} s \mathrm{Posin} \alpha_{p} e^{\mathrm{i} \phi}, \\
\nabla \cdot \widehat{\boldsymbol{u}}=0,
\end{gathered}
$$

subject to the influx condition from the boundary layer. The second set governs the boundary-layer flow $(\widetilde{\boldsymbol{u}}, \widetilde{p})$,

$$
\begin{aligned}
\mathrm{i} \tilde{\boldsymbol{u}}+2 \hat{z} \times \tilde{\boldsymbol{u}}+(\hat{\boldsymbol{n}} \cdot \nabla \tilde{p}) \hat{\boldsymbol{n}} & =E(\hat{\boldsymbol{n}} \cdot \nabla \widetilde{\boldsymbol{u}})^{2}, \\
0 & =\nabla \cdot \widetilde{\boldsymbol{u}},
\end{aligned}
$$

where $\hat{\boldsymbol{n}}$ denotes unit normal to the bounding surface $\mathcal{S}$ of the cylinder. The no-slip condition is obeyed by the sum of the boundary flow $\tilde{\boldsymbol{u}}$ and the mainstream flow $\mathcal{A} \boldsymbol{u}_{112}$.

The inhomogeneous system (13) and (14) requires a solvability condition which reads

$$
\begin{gathered}
\left(4 \pi^{2} \mathcal{A}\right) E \int_{0}^{1} \int_{0}^{\Gamma} \int_{0}^{2 \pi}\left|\boldsymbol{u}_{112}\right|^{2} s \mathrm{~d} \phi \mathrm{d} s \mathrm{~d} z+\int_{\mathcal{S}} p_{112}^{*}(\hat{\boldsymbol{n}} \cdot \widehat{\boldsymbol{u}}) \mathrm{d} \mathcal{S} \\
=-2 \operatorname{Po} \sin \alpha_{p} \int_{0}^{1} \int_{0}^{\Gamma} \int_{0}^{2 \pi}\left(\hat{z} \cdot \boldsymbol{u}_{112}^{*}\right) e^{\mathrm{i} \phi} s^{2} \mathrm{~d} \phi \mathrm{d} s \mathrm{~d} z,
\end{gathered}
$$

where $f^{*}$ denotes the complex conjugate of $f$. In the solvability condition, the surface integral, $\int_{\mathcal{S}} p_{112}^{*}(\hat{\boldsymbol{n}} \cdot \widehat{\boldsymbol{u}}) \mathrm{d} \mathcal{S}$, represents the influx from the viscous boundary layer $\tilde{\boldsymbol{u}}$, which can be readily evaluated. After carrying out all the integrals in the solvability condition, (15), we obtain an equation for determining the amplitude $\mathcal{A}$ of the precessing flow, which is

$$
\mathcal{A}=\left(\frac{\operatorname{Pos} \sin \alpha_{p}}{\sqrt{E}}\right)\left[\frac{\mathrm{i} 164.77}{(37578 \sqrt{E}+2248.8)+\mathrm{i} 735.33}\right] .
$$

Inserting the amplitude $\mathcal{A}$ and the boundary-layer flow $\tilde{\boldsymbol{u}}$ into the asymptotic expansion, (11), gives rise to an analytical solution in closed form describing the weakly precessing flow,

$$
\begin{aligned}
\boldsymbol{u}(s, \phi, z, t)= & \frac{\mathcal{A}}{2}\left\{-\left(\boldsymbol{u}_{112}-\mathrm{i} \hat{\boldsymbol{z}} \times \boldsymbol{u}_{112}\right)_{z=0} e^{-\sqrt{3}(1+\mathrm{i}) z / \sqrt{2 E}}\right. \\
& -\left(\boldsymbol{u}_{112}+\mathrm{i} \hat{z} \times \boldsymbol{u}_{112}\right)_{z=0} e^{-(1-\mathrm{i}) z / \sqrt{2 E}} \\
& -\left(\boldsymbol{u}_{112}-\mathrm{i} \hat{z} \times \boldsymbol{u}_{112}\right)_{z=1} e^{-\sqrt{3}(1+\mathrm{i})(1-z) / \sqrt{2 E}} \\
& -\left(\boldsymbol{u}_{112}+\mathrm{i} \hat{z} \times \boldsymbol{u}_{112}\right)_{z=1} e^{-(1-\mathrm{i})(1-z) / \sqrt{2 E}} \\
& \left.+2 \boldsymbol{u}_{112}-\left(2 \boldsymbol{u}_{112}\right)_{s=\Gamma_{2}} e^{-(1+\mathrm{i})(\Gamma-s) / \sqrt{2 E}}\right\} e^{\mathrm{i} t}
\end{aligned}
$$

which is valid for $0<E \ll 1$ and an arbitrary angle $\alpha_{p}$ in the weakly precessing cylinder with $\Gamma=\Gamma_{2}$. Based on the analytical expression, (16), we can readily calculate its kinetic energy density $E_{\text {kin }}$,

$$
\begin{aligned}
E_{\text {kin }} & =\frac{1}{2\left(\pi \Gamma^{2}\right)} \int_{0}^{1} \int_{0}^{\Gamma} \int_{0}^{2 \pi}|\boldsymbol{u}|^{2} s \mathrm{~d} \phi \mathrm{d} s \mathrm{~d} z \\
& =\left[\frac{2\left(\Gamma_{2} \pi\right)^{2}+1}{6\left(\Gamma_{2}\right)^{2}}\right]|\mathcal{A}|^{2} J_{1}^{2}(5.6914),
\end{aligned}
$$

which not only measures the strength of the precessing flow but also can be employed to check with the corresponding numerical solution.

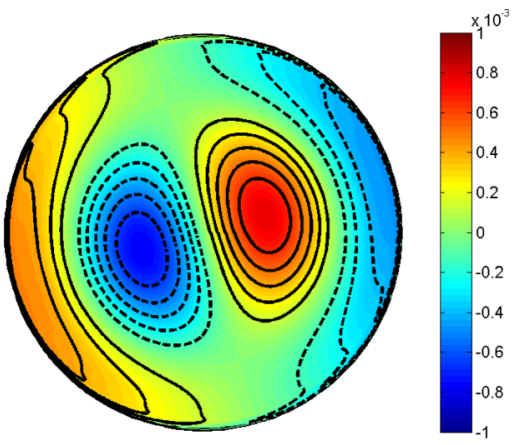

(a)

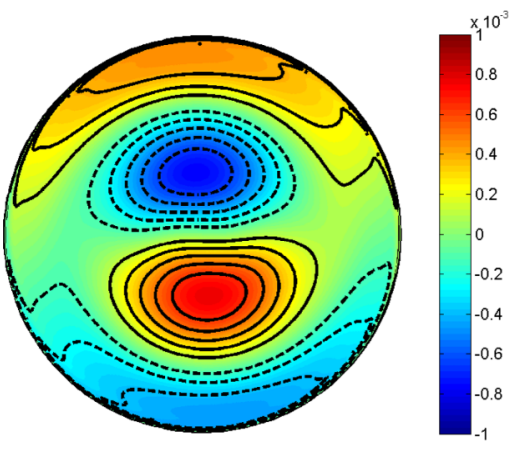

(b)

FIG. 1. (Color online) Contours of $u_{z}$ of precessing flow at an instant in the mantle frame at the $z=1 / 2$ plane for $\mathrm{Po}=10^{-4}$ and $E=10^{-4}$ in the precessing cylinder with $\Gamma=\Gamma_{2}$ : (a) analytical solution computed from expression (16) and (b) corresponding finite-element solution from nonlinear numerical simulation. It should be noted that, since both solutions are in the form of an azimuthally traveling wave, the difference in the azimuthal phase between (a) and (b) has no mathematical or physical significance. 


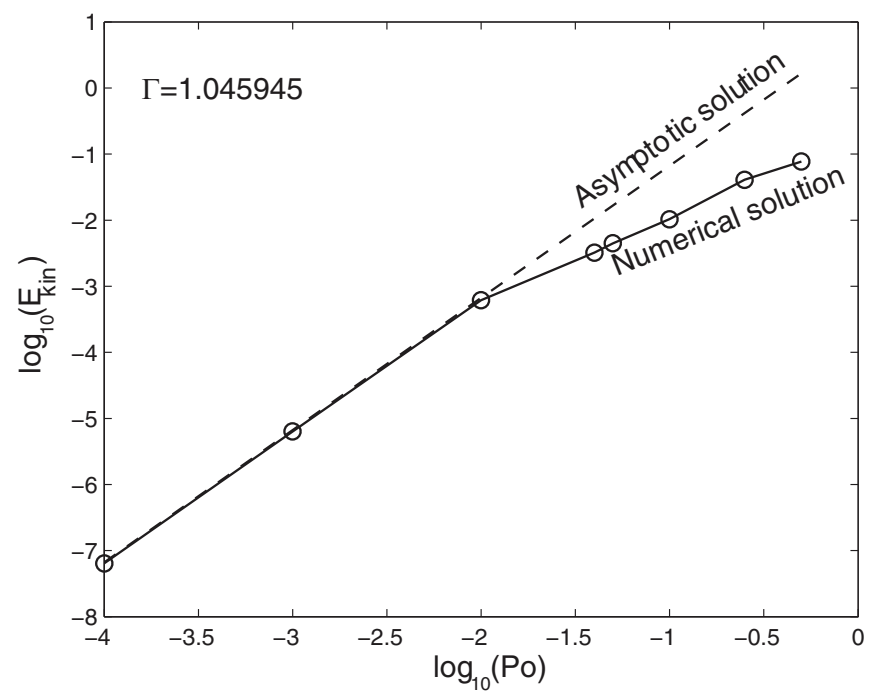

FIG. 2. Kinetic energy densities $E_{\text {kin }}$ as a function of Po for $\alpha_{p}=\pi / 4$ and $E=10^{-4}$ at the second resonance in the precessing cylinder with aspect ratio $\Gamma=\Gamma_{2}$. The asymptotic solution (dashed line) is computed from the analytical expression, (17), while circles represent the solutions of finite element simulation.

Figure 1(a) depicts the flow structure computed from expression (16) with $E=10^{-4}$, Po $=10^{-4}$, and $\alpha_{p}=\pi / 4$ in a drifting frame in which the precessing flow is steady. Apart from the key feature that its amplitude $|\boldsymbol{u}|=O(\mathrm{Po} / \sqrt{E})$, there exists a zero nodal surface in the precessing flow at $s \approx 0.6$, which, as we show, will have important nonlinear consequences. Figure 2 presents the kinetic energy density $E_{\text {kin }}$ as a function of Po computed from the analytical formula, (17), for $E=10^{-4}$ and $\alpha_{p}=\pi / 4$. The corresponding numerical solutions are also shown in Figs. 1 and 2, which are discussed in the next section.

\section{NONLINEAR FLOW AT THE SECOND AND THIRD RESONANCES}

\section{A. A finite-element method in the tetrahedralized cylinder}

We tackle the nonlinear problem via direct numerical simulation using a three-dimensional finite-element method based on the tetrahedralization of the entire cylindrical cavity. This method allows us to extend the weakly precessing regime $(\mathrm{Po} / \sqrt{E}<1)$ to the strongly precessing regime $[\mathrm{Po} / \sqrt{E} \geqslant$ $O(1)]$. Since the detail of the finite-element code, its validation against a constructed exact solution, and its convergence properties have been discussed in [23](see also [24] and [25]), this section is brief.

A major advantage of the finite-element method is that it does not have axial singularities and is flexible enough to construct dense nodes in the vicinity of the bounding surface of the cylindrical cavity for resolving the thin viscous boundary layer. Let $T_{f}$ be the fixed final time of a numerical simulation. We then divide the time interval $\left[0, T_{f}\right]$ into $M$ equally spaced subintervals using the nodal points

$$
0=t_{0}<t_{1}<t_{2}<\cdots<t_{M}=T_{f},
$$

where $t_{n}=n \Delta t$ for $n=0,1, \ldots, M$. Let $\boldsymbol{u}(\mathbf{r}, t)$ be a function continuous with respect to $t$, where $\mathbf{r}$ is the position vector. Denote $\boldsymbol{u}^{n}(\mathbf{r})=\boldsymbol{u}\left(\mathbf{r}, t_{n}\right)$ for $n=0,1, \ldots, M$. An implicit time stepping scheme is employed for the time advancement of numerical integration in which an implicit second-order backward differentiation formula is adopted for the time derivative

$$
\left(\frac{\partial \boldsymbol{u}}{\partial t}\right)^{n+1}=\frac{3 \boldsymbol{u}^{n+1}-4 \boldsymbol{u}^{n}+\boldsymbol{u}^{n-1}}{2 \Delta t}+O\left(\Delta t^{2}\right),
$$

while the nonlinear term $\boldsymbol{u} \cdot \nabla \boldsymbol{u}$ at $t=t_{n+1}$ is approximated by the implicit scheme

$$
\boldsymbol{u}^{n+1} \cdot \nabla \boldsymbol{u}^{n+1}=\left(2 \boldsymbol{u}^{n}-\boldsymbol{u}^{n-1}\right) \cdot \nabla \boldsymbol{u}^{n+1}+O\left(\Delta t^{2}\right) .
$$

The implicit temporal discretization of the full governing equations, (1) and (2), yields

$$
\begin{gathered}
\frac{3 \boldsymbol{u}^{n+1}-4 \boldsymbol{u}^{n}+\boldsymbol{u}^{n-1}}{2 \Delta t}+\left(2 \boldsymbol{u}^{n}-\boldsymbol{u}^{n-1}\right) \cdot \nabla \boldsymbol{u}^{n+1} \\
+2\left(\hat{z}+\operatorname{Po} \widehat{\boldsymbol{\Omega}}_{p}^{n+1}\right) \times \boldsymbol{u}^{n+1}+\nabla p^{n+1} \\
=E \nabla^{2} \boldsymbol{u}^{n+1}-2 \operatorname{Po}\left[s \sin \alpha_{p} \cos \left(\phi+t_{n+1}\right)\right] \hat{z}, \\
\nabla \cdot \boldsymbol{u}^{n+1}=0,
\end{gathered}
$$

where

$\widehat{\boldsymbol{\Omega}}_{p}^{n+1}=\sin \alpha_{p}\left[\hat{\boldsymbol{s}} \cos \left(\phi+t_{n+1}\right)-\hat{\boldsymbol{\phi}} \sin \left(\phi+t_{n+1}\right)\right]+\hat{z} \cos \alpha_{p}$.

They are solved, starting from an arbitrary initial condition, to find $\boldsymbol{u}^{n+1}$ and $p^{n+1}$ for a given $\boldsymbol{u}^{n}$ and $p^{n}$, subject to the boundary conditions, (3) and (4), on modern parallel computers.

The nonlinear cylindrical precession code was carefully validated against a constructed exact solution [23], showing excellent agreement between the computed nonlinear numerical solution and the exact solution. We have adopted a nonuniform three-dimensional mesh that is marked by more nodes in the vicinity of the bounding surface of the cylindrical cavity for resolving the thin viscous boundary layer. The typical finite-element size $h$ in the boundary-layer region of the three-dimensional mesh is chosen to be of the order $\sqrt{E}$. For example, the three-dimensional mesh in the case $\Gamma=1.611089$ contains about $2.7 \times 10^{6}$ tetrahedral elements along with $10.6 \times 10^{6}$ unknowns. The size of $T_{f}$ selected for direct numerical simulation depends mainly on the size of $E$. Since the precessing flow at nonlinear equilibrium at direct resonance is dynamically controlled by the effect of the viscous boundary layer, sufficiently long integration, typically with $T_{f} \geqslant O(1 / \sqrt{E})$ at $0<\sqrt{E} \ll 1$, is required.

\section{B. Comparison with the analytical solution}

The form of the analytical solution, (16), suggests that, in order to understand the properties of precessing flow, we need to know which/how inertial modes are excited and sustained by the effect of precession. It follows that an effective way of comparing a numerical solution to the analytical solution, as well as capturing the nature of temperospatially complicated flow, is to decompose the precessing flow $\boldsymbol{u}$ into the complete 
set of cylindrical inertial modes [26]

$$
\begin{aligned}
\boldsymbol{u}= & \tilde{\boldsymbol{u}}+\sum_{k=1}^{K} \mathcal{A}_{00 k}(t) \boldsymbol{u}_{00 k}(s) \\
& +\sum_{m=1}^{M} \sum_{k=1}^{K} \frac{1}{2}\left[\mathcal{A}_{m 0 k}(t) \boldsymbol{u}_{m 0 k}(s, \phi)+\text { c.c. }\right] \\
& +\sum_{n=1}^{N} \sum_{k=1}^{K} \frac{1}{2}\left[\mathcal{A}_{0 n k}(t) \boldsymbol{u}_{0 n k}(s, z)+\text { c.c. }\right] \\
& +\sum_{m=1}^{M} \sum_{n=1}^{N} \sum_{k=1}^{2 K} \frac{1}{2}\left[\mathcal{A}_{m n k}(t) \boldsymbol{u}_{m n k}(s, z, \phi)+\text { c.c. }\right],
\end{aligned}
$$

where c.c. denotes the complex conjugate of the preceding term, $\tilde{\boldsymbol{u}}$ represents the boundary-layer flow, $\boldsymbol{u}_{00 k}$ and $\boldsymbol{u}_{m 0 k}$ with $m \geqslant 1$ and $k \geqslant 1$ represent the axisymmetric and nonaxisymmetric geostrophic mode, respectively, $\boldsymbol{u}_{0 n k}$ with $n \geqslant 1$ and $k \geqslant 1$ are the axisymmetric inertial modes, and $\boldsymbol{u}_{m n k}$ with $m \geqslant 1, n \geqslant 1$, and $k \geqslant 1$ denote the nonaxisymmetric inertial wave modes. The inertial modes are normalized to satisfy the orthonormal condition

$$
\int_{0}^{1} \int_{0}^{\Gamma} \int_{0}^{2 \pi}\left(\boldsymbol{u}_{m n k}^{*} \cdot \boldsymbol{u}_{m n k}\right) s \mathrm{~d} \phi \mathrm{d} s \mathrm{~d} z=1
$$

for all possible $m, n$, and $k$. For the parameter range discussed in this paper, the truncation with $M \leqslant 10, N \leqslant 10$, and $K \leqslant 10$ in (20) seems sufficient. The general expressions for $\boldsymbol{u}_{m n k}$ and $p_{m n k}$ can be found, for example, in [18-20]. It is also convenient to define the amplitude $\mathcal{A}_{G}$ for axisymmetric geostrophic flow as

$$
\mathcal{A}_{G}=\sqrt{\left[\sum_{k=1}^{K}\left|\mathcal{A}_{00 k}\right|^{2}\right]} .
$$

In addition to the total kinetic energy density $E_{\text {kin }}$, we also introduce the kinetic energy density for the axisymmetric geostrophic component

$$
\begin{aligned}
E_{\mathrm{geo}} & =\frac{1}{2 \pi \Gamma^{2}} \int_{0}^{1} \int_{0}^{\Gamma} \int_{0}^{2 \pi}\left|U_{G} \hat{\boldsymbol{\phi}}\right|^{2} s \mathrm{~d} \phi \mathrm{d} s \mathrm{~d} z \\
& =\frac{1}{2 \pi \Gamma^{2}} \sum_{k=1}^{K}\left|\mathcal{A}_{00 k}\right|^{2},
\end{aligned}
$$

where $U_{G} \hat{\boldsymbol{\phi}}$ represents the axisymmetric geostrophic flow $\left(\partial U_{G} / \partial z=0, \partial U_{G} / \partial \phi=0\right)$, and the kinetic energy density for the resonant inertial mode $\boldsymbol{u}_{11 k}$

$$
\begin{aligned}
E_{11 \mathrm{k}} & =\frac{1}{2 \pi \Gamma^{2}} \int_{0}^{1} \int_{0}^{\Gamma} \int_{0}^{2 \pi}\left|\mathcal{A}_{11 k} \boldsymbol{u}_{11 k}\right|^{2} s \mathrm{~d} \phi \mathrm{d} s \mathrm{~d} z \\
& =\frac{1}{2 \pi \Gamma^{2}}\left(\frac{\left|\mathcal{A}_{11 k}(t)\right|^{2}}{2}\right),
\end{aligned}
$$

where $k=2$ corresponds to the cylinder with $\Gamma=\Gamma_{2}$, and $k=3$ to the cylinder with $\Gamma=\Gamma_{3}$.

Displayed in Fig. 1(b) is the structure of the weakly precessing flow, plotted using the solution from direct nonlinear numerical simulation, for $\alpha_{p}=\pi / 4, \mathrm{Po}=10^{-4}$, and $E=10^{-4}$ in the precessing cylinder with $\Gamma=\Gamma_{2}$. There exist, comparing Fig. 1(b) to the analytical solution in Fig. 1(a), no significant differences between the analytical solution, (17), and the corresponding numerical solution. This is because nonlinear effects in the case of $\mathrm{Po}=10^{-4}$ and $E=10^{-4}$, along with $\mathrm{Po} / \sqrt{E}=0.01$, are quite weak, and consequently, the contribution from $\mathcal{A}_{112} \boldsymbol{u}_{112}$ in the expansion, (20), is highly dominant, consistent with the assumption/expansion used in our asymptotic analysis.

When $\mathrm{Po} / \sqrt{E}<O(1)$, it is anticipated that the leadingorder behavior would be approximately described by analytical expressions (16) and (17). Referring to the results plotted in Fig. 2, it is clear that the asymptotic solution given by (16) and (17) provides a good approximation when $\mathrm{Po} / \sqrt{E} \leqslant 1$. Figure 2 shows that, in the parameter range $0<\mathrm{Po} / \sqrt{E} \leqslant 1$, where the precessing flow is laminar with a constant amplitude (i.e., the kinetic energy density $E_{\text {kin }}$ is independent of time), a good agreement is achieved between the asymptotic solution [dashed line computed from (17) shown in Fig. 2] and the nonlinear numerical solutions (represented by circles in Fig. 2). However, our main concern in this study is how the nature of turbulence is controlled by an axisymmetric geostrophic flow in the parameter range $\mathrm{Po} / \sqrt{E}>1$.

\section{Nonlinear properties at the second resonance}

At the heart of understanding nonlinear properties is the axisymmetric geostrophic flow in the form of the differential rotation $U_{G}(s) \hat{\boldsymbol{\phi}}$. It will be seen that the geostrophic flow $U_{G}(s) \hat{\boldsymbol{\phi}}$ would be capable of controlling the nature of turbulence in the precessing cylinder whose fluid motion is at direct resonance with the Poincaré force.

Prior to discussing the details of nonlinear solutions, it should be noted that the axisymmetric, steady geostrophic flow $U_{G}(s) \hat{\boldsymbol{\phi}}$ cannot be generated/maintained by the nonlinear interaction of the resonant inertial mode $\boldsymbol{u}_{112}$ in the interior of the cylinder. This is because the Reynolds stress associated

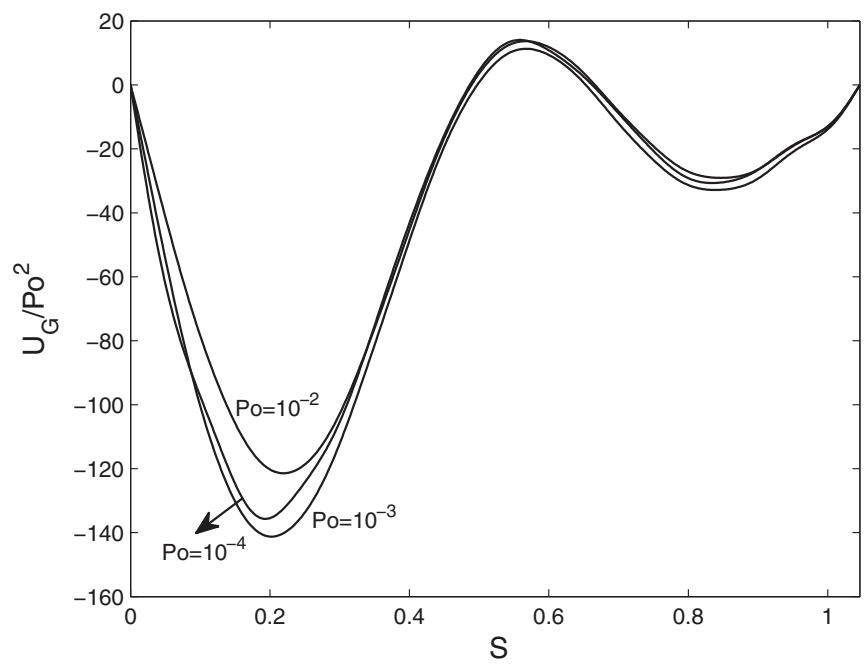

FIG. 3. Scaled axisymmetric, steady geostrophic flow $U_{G}(s) / \mathrm{Po}^{2}$ as a function of $s$ for $\mathrm{Po}=10^{-4}, 10^{-3}$, and $10^{-2}$ at $E=10^{-4}$ in the precessing cylinder with aspect ratio $\Gamma=\Gamma_{2}$. 


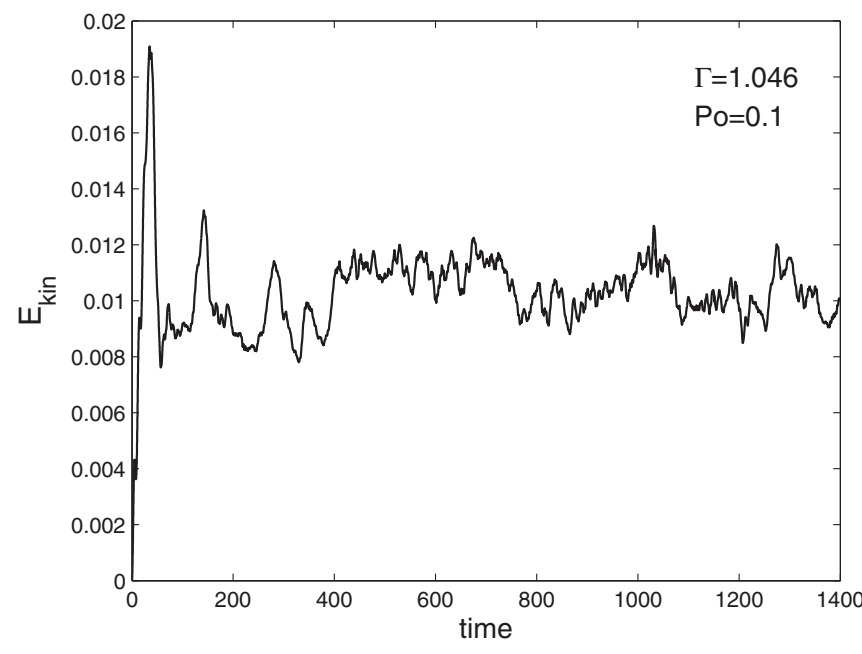

FIG. 4. Kinetic energy densities $E_{\text {kin }}$ of the precessing flow as a function of time for $\alpha_{p}=\pi / 4$ at $E=10^{-4}$ in the resonant cylinder with $\Gamma=\Gamma_{2}$ for Po $=0.1$. Direct numerical simulation lasts about 223 revolutions.

with $\boldsymbol{u}_{112}$ in the interior vanishes identically,

$$
\int_{0}^{2 \pi} \int_{0}^{1}\left(\mathcal{A}_{112} \boldsymbol{u}_{112}+\text { c.c. }\right) \cdot \nabla\left(\mathcal{A}_{112} \boldsymbol{u}_{112}+\text { c.c. }\right) \mathrm{d} z \mathrm{~d} \phi=\mathbf{0},
$$

where $\boldsymbol{u}_{112}$ is given by (8)-(10). It is the nonlinear effect within the viscous boundary layer that induces the steady geostrophic flow $U_{G}(s) \hat{\boldsymbol{\phi}}$. For the purpose of illustrating how $U_{G}(s) \hat{\boldsymbol{\phi}}$ is generated, we write the leading-order boundary flow at the bottom in the form

$$
\tilde{\boldsymbol{u}}=\frac{\mathrm{Po}}{\sqrt{E}}\left\{\hat{\boldsymbol{s}}\left[f(s) e^{-(1-\mathrm{i}) z / \sqrt{2 E}} e^{\mathrm{i}(\phi+t)}+\text { c.c. }\right]+\cdots\right\},
$$

where $f(s)$ is a real function. It is important to note that the axisymmetric Reynolds stress in the boundary layer,

$\frac{1}{2 \pi} \int_{0}^{2 \pi} \widetilde{\boldsymbol{u}} \times \nabla \times \widetilde{\boldsymbol{u}} \mathrm{d} \phi=\left(\frac{\mathrm{Po}}{\sqrt{E}}\right)^{2}\left[\hat{\boldsymbol{s}} f(s) \frac{\mathrm{d} f}{\mathrm{~d} s} e^{-2 z / \sqrt{E}}+\cdots\right]$,

does not vanish, is time independent, and can induce the steady geostrophic flow $U_{G}(s) \hat{\boldsymbol{\phi}}$ in the interior at the order $(\mathrm{Po} / \sqrt{E})^{2}$ (see, for example, [5]).

Figure 3 shows the profile of the axisymmetric, steady geostrophic flow $U_{G}(s)$ for three values of the Poincaré number, $\mathrm{Po}=10^{-4}, 10^{-3}$, and $10^{-2}$ at $E=10^{-4}$ in the precessing cylinder with $\Gamma=\Gamma_{2}$. A profound difference should be noted between the fundamental resonance at $\Gamma=\Gamma_{1}$ and the second resonance at $\Gamma=\Gamma_{2}$. The geostrophic flow $U_{G}(s)$ in the case $\Gamma=\Gamma_{1}$ is always retrograde in the whole cylinder $\left[U_{G}(s)<0,0<s<\Gamma\right]$ [22], while the geostrophic flow $U_{G}(s)$ in the case $\Gamma=\Gamma_{2}$ is in the form of the differential rotation, which can be, depending on the distance $s$ from the rotation axis, either retrograde $U_{G}(s)<0$ or prograde $U_{G}(s)>0$. Spatially, the geostrophic flow structure $U_{G}(s)$ reflects the profile of the boundary-layer flow $\tilde{\boldsymbol{u}}$ at the top and bottom of the cylinder, which is determined by the structure of the resonant inertial mode $\boldsymbol{u}_{112}$. Temporally, the axisymmetric geostrophic flow $U_{G}(s) \hat{\boldsymbol{\phi}}$ in Fig. 3 is stationary because the resonant inertial mode $\boldsymbol{u}_{112}$, given by (8)-(10), represents an azimuthally traveling wave.
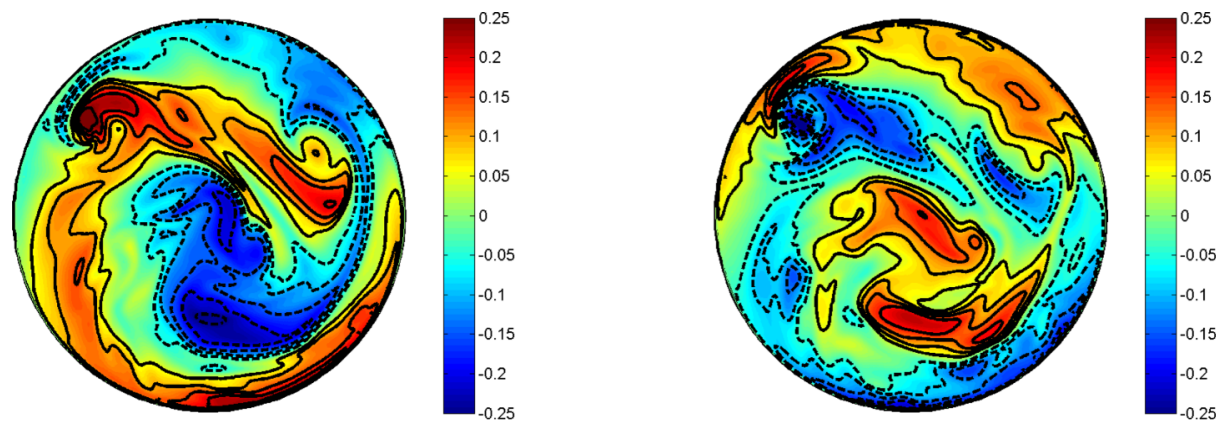

(a)

(b)
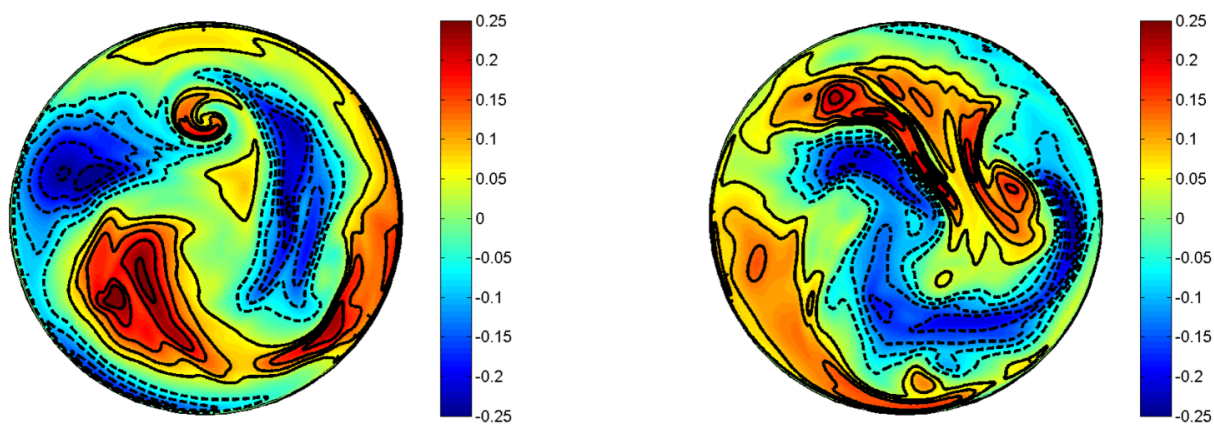

(c)

(d)

FIG. 5. (Color online) Contours of $u_{z}$ of strongly turbulent precessing flow with highly irregular fluctuation at four instants on the $z=1 / 2$ plane for Po $=0.1$ and $E=10^{-4}$ in the precessing cylinder with $\Gamma=\Gamma_{2}$. 


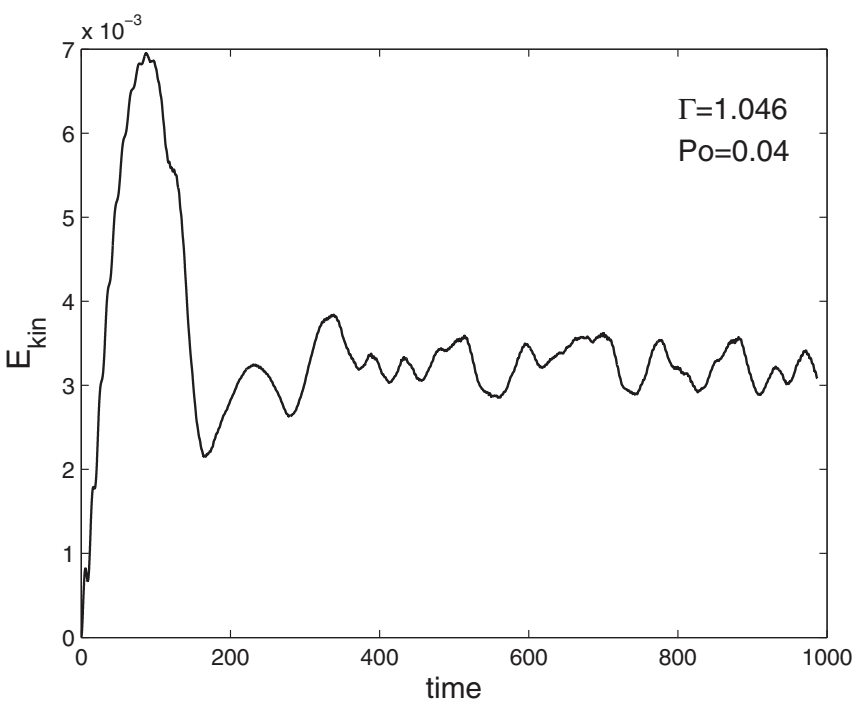

FIG. 6. Kinetic energy densities $E_{\text {kin }}$ of strongly turbulent precessing flow as a function of time for Po $=0.04$ and $E=10^{-4}$ in the resonant cylinder with aspect ratio $\Gamma=\Gamma_{2}$.

While the nonlinear precessing flow in the parameter range $0<\mathrm{Po} / \sqrt{E} \leqslant 1$ is laminar with a constant amplitude, the flow, marked by its highly irregular temporal-spatial fluctuation, becomes strongly turbulent in the approximate range $1<(\mathrm{Po} / \sqrt{E}) \leqslant 10$. As an example of strong turbulence, the kinetic energy densities $E_{\text {kin }}(t)$ and the flow profiles at several instants are shown in Figs. 4 and 5 for $\mathrm{Po}=0.1$ and $E=10^{-4}$ with $\Gamma=\Gamma_{2}$. Important questions are why and how
TABLE I. Largest five coefficients $\left|A_{m n k}\right|$ of the precessing flow with $\alpha_{p}=\pi / 4, \Gamma=\Gamma_{2}$, and $\mathrm{Po}=0.04$ for $E=10^{-4}$ after about 13 revolutions at $t=85.4$. The superscript $(m, n, k)^{-}$denotes an inertial mode with a negative frequency.

\begin{tabular}{lcc}
\hline \hline$(m, n, k)$ & $\left|\mathcal{A}_{m n k}\right|$ & $\sigma_{m n k}$ \\
\hline$(1,1,2)$ & $2.7540 \times 10^{-1}$ & +0.5000 \\
$(1,1,1)$ & $5.2215 \times 10^{-2}$ & +0.7952 \\
$(1,1,3)$ & $5.1310 \times 10^{-2}$ & +0.3477 \\
$(1,1,2)^{-}$ & $2.2796 \times 10^{-2}$ & -0.3711 \\
$(0,2,1)$ & $2.1563 \times 10^{-2}$ & +0.8639 \\
\hline \hline
\end{tabular}

the precessing flow becomes strongly turbulent. We attempt to answer these questions by performing a careful numerical experiment at $\mathrm{Po}=0.04$ and $E=10^{-4}$ with $\Gamma=\Gamma_{2}$. In this numerical experiment, we suddenly turn on precession at $t=0$ with $\boldsymbol{u}=0$ in the mantle frame of reference and, then, finish the experiment at $T_{f}=10 / \sqrt{E}$ after about 160 revolutions when the system reaches nonlinear equilibrium. The time evolution of the numerical experiment is illustrated in Figs. 6-8. It should be noted that we can accurately decompose the precessing flow at any instants into the complete spectral space of cylindrical inertial modes and, hence, offer a helpful way of understanding its nonlinear evolution. During the initial period of the experiment, $0<t<O(1 / \sqrt{E})$, the inertial mode $\boldsymbol{u}_{112}$ resonates directly with the precessing force and the amplitude $\left|\mathcal{A}_{112}\right|$ increases rapidly with time. After about 13 revolutions at $t=85.4$, the amplitude of the resonant mode $\boldsymbol{u}_{112}$ reaches its peak value at $\left|\mathcal{A}_{112}\right|=0.2754$, which is, as illustrated in Table I, predominant over nonresonant inertial modes. It is

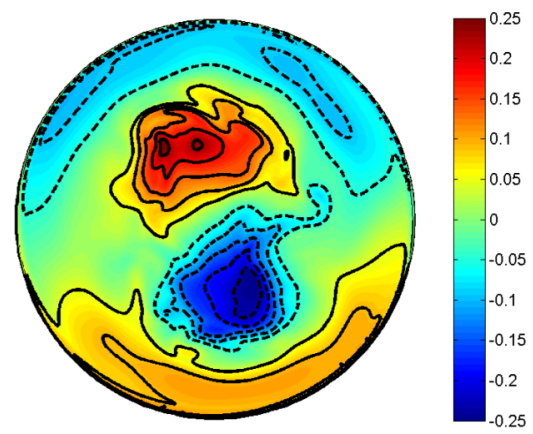

(a)

(b)
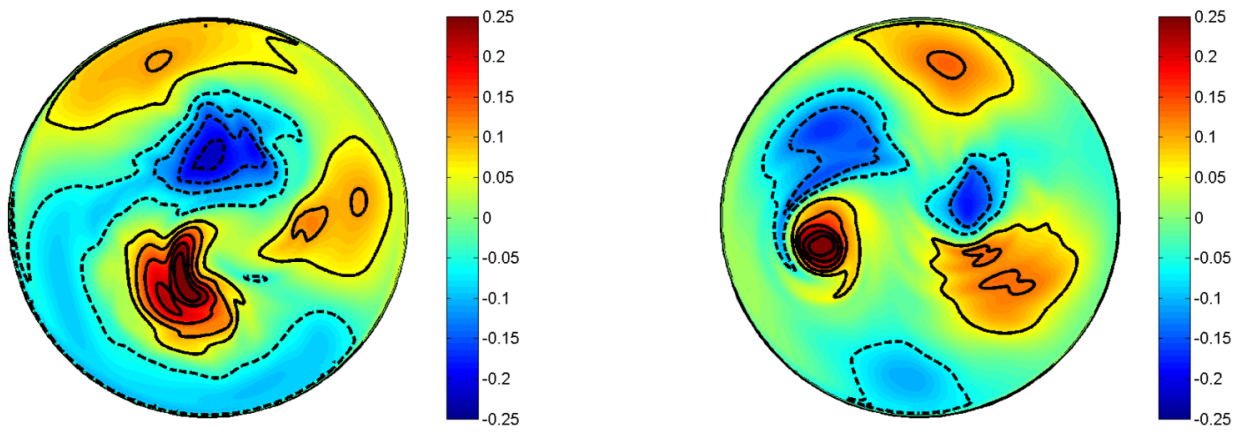

(c) (d)

FIG. 7. (Color online) Contours of $u_{z}$ in the mantle frame on the $z=1 / 2$ plane for Po $=0.04$ and $E=10^{-4}$ in the precessing cylinder with $\Gamma=\Gamma_{2}$ at four instants, $t=85,112,127$, and 140, during its transition to turbulence. 

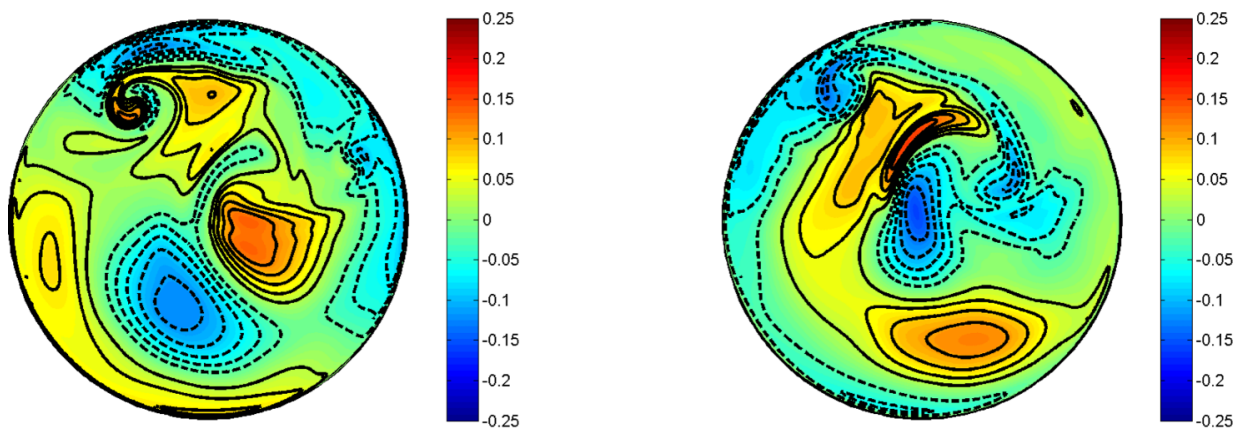

(a)

(b)
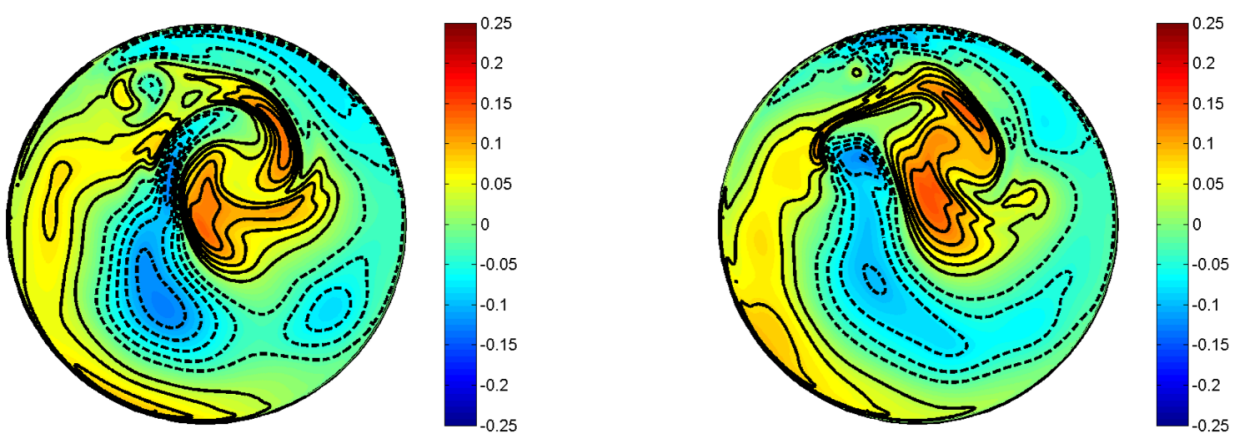

(c)

(d)

FIG. 8. (Color online) Contours of $u_{z}$ in the mantle frame on the $z=1 / 2$ plane for Po $=0.04$ and $E=10^{-4}$ in the precessing cylinder with $\Gamma=\Gamma_{2}$ at four instants after reaching nonlinear equilibrium: (a) $t=979$, (b) $t=990$, (c) $t=998.5$, and (d) $t=1000$.

crucial to note that many nonresonant inertial modes, driven by the boundary-layer influx, exist but are weak and, hence, insignificant during this initial period. When $t=O(1 / \sqrt{E})$, nonresonant inertial modes become sufficiently strong to be able to interact with the resonant mode $\boldsymbol{u}_{112}$, leading to the strongly turbulent flow. However, strong turbulence at the second resonance at $\Gamma=\Gamma_{2}$ is not caused by the mechanism of triadic resonance, which is primarily involved in the three inertial modes: the resonant mode $\boldsymbol{u}_{112}$ with the half-frequency $\sigma_{112}=1 / 2$ and two unforced inertial modes, $\boldsymbol{u}_{\tilde{m} \tilde{n} \tilde{k}}$ with $\sigma_{\tilde{m} \tilde{n} \tilde{k}}$

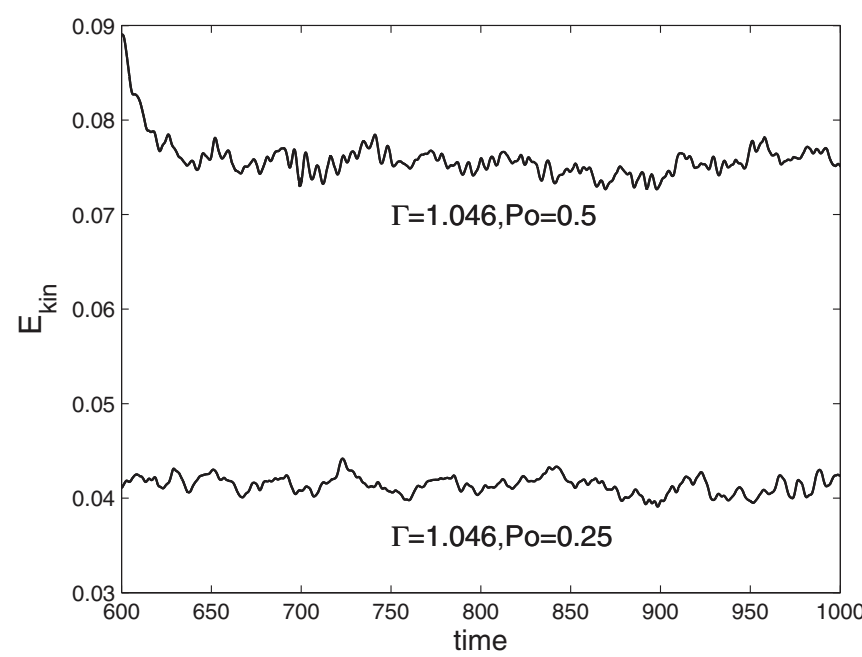

FIG. 9. Kinetic energy densities $E_{\text {kin }}$ of the weakly turbulent flow as a function of time for $E=10^{-4}$ in the resonant cylinder with $\Gamma=\Gamma_{2}$ for $\mathrm{Po}=0.25$ and $\mathrm{Po}=0.5$. and $\boldsymbol{u}_{\hat{m} \hat{n} \hat{k}}$ with $\sigma_{\hat{m} \hat{n} \hat{k}}$, which satisfy the parametric conditions

$$
|\tilde{m}-\hat{m}|=1, \quad|\tilde{n}-\hat{n}|=1, \quad\left|\sigma_{\tilde{m} \tilde{n} \tilde{k}}-\sigma_{\hat{m} \hat{n} \hat{k}}\right|=\frac{1}{2} .
$$

Table II lists the largest five coefficients $\left|A_{m n k}\right|$ of the nonlinear flow at $t=139.8$ after about 22 revolutions when the flow becomes strongly turbulent. It can be seen that the inertial mode $\boldsymbol{u}_{311}$ with its frequency $\sigma_{311}=0.5104$ becomes predominant at that instant but two other inertial modes to satisfy the condition of triadic resonance are absent. After several spinning-up time scales when the precessing flow becomes strongly turbulent and attains its nonlinear equilibrium, it is found that the coefficient $\left|A_{112}\right|$ is always the largest but there also exist a large number of nonresonant inertial modes, such as $\boldsymbol{u}_{012}$ and $\boldsymbol{u}_{211}$, that are excited by both nonlinear and viscous effects and that are not unsubstantial. A typical spectrum of the largest 10 coefficients $\left|A_{m n k}\right|$ at nonlinear equilibrium is presented in Table III after about 158 revolutions at $t=990$, showing that the resonant mode $\left|A_{112}\right|=0.096$ along with many nonresonant modes whose amplitudes have

TABLE II. Largest five coefficients $\left|A_{m n k}\right|$ of the precessing flow with $\alpha_{p}=\pi / 4, \Gamma=\Gamma_{2}$, and $\mathrm{Po}=0.04$ for $E=10^{-4}$ after about 22 revolutions at $t=139.8$.

\begin{tabular}{lcc}
\hline \hline$(m, n, k)$ & $\left|\mathcal{A}_{m n k}\right|$ & $\sigma_{m n k}$ \\
\hline$(3,1,1)$ & $1.1724 \times 10^{-1}$ & +0.5104 \\
$(1,1,2)$ & $8.3083 \times 10^{-2}$ & +0.5000 \\
$(2,0,1)$ & $6.4888 \times 10^{-2}$ & +0.0000 \\
$(1,1,1)$ & $5.7395 \times 10^{-2}$ & +0.7952 \\
$(2,0,2)$ & $5.5164 \times 10^{-2}$ & +0.0000 \\
\hline
\end{tabular}


TABLE III. Largest 10 coefficients $\left|A_{m n k}\right|$ of the strongly turbulent flow with $\Gamma=\Gamma_{2}$ and Po $=0.04$ for $E=10^{-4}$ at nonlinear equilibrium after about 158 revolutions at $t=990$.

\begin{tabular}{lcc}
\hline \hline$(m, n, k)$ & $\left|\mathcal{A}_{m n k}\right|$ & $\sigma_{m n k}$ \\
\hline$(1,1,2)$ & $9.5784 \times 10^{-2}$ & +0.5000 \\
$(1,0,1)$ & $5.9660 \times 10^{-2}$ & +0.0000 \\
$(0,1,2)$ & $5.9350 \times 10^{-2}$ & +0.4242 \\
$(2,1,1)^{-}$ & $5.2670 \times 10^{-2}$ & -0.4759 \\
$(1,0,2)$ & $5.2614 \times 10^{-2}$ & +0.0000 \\
$(1,1,1)$ & $4.5362 \times 10^{-2}$ & +0.7952 \\
$(2,0,1)$ & $4.4595 \times 10^{-2}$ & +0.0000 \\
$(1,1,3)$ & $4.3658 \times 10^{-2}$ & +0.3477 \\
$(3,1,1)$ & $3.9819 \times 10^{-2}$ & +0.5104 \\
$(1,1,1)^{-}$ & $3.4968 \times 10^{-2}$ & -0.5507 \\
\hline \hline
\end{tabular}

the same order of magnitude. Note that the axisymmetric geostrophic flow whose amplitude is $\mathcal{A}_{G}=0.02894$ is weak and, hence, not listed in Table III. Although the resonant mode $\boldsymbol{u}_{112}$ is always prominent at nonlinear equilibrium after $t>O(1 / \sqrt{E})$, we are unable, despite a significant effort to search for, to identify the two free inertial modes at any instants that are substantial and can form triadic resonance with the resonant mode $\boldsymbol{u}_{112}$. There is no indication that a triadic resonant instability would break the symmetry of the laminar precessing flow and, then, lead to the strongly turbulent flow. Moreover, the nonlinearly saturated state in the equilibrium state is strongly insensitive to the initial conditions.

Our numerical experiments in the approximate range $1<(\mathrm{Po} / \sqrt{E}) \leqslant 10$ suggest that relatively simple nonlinear mechanisms describe the large-scale behavior of strongly

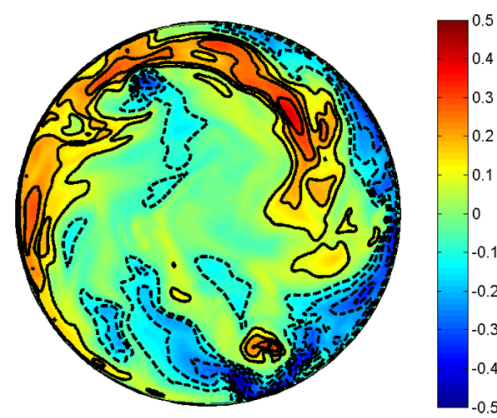

(a)

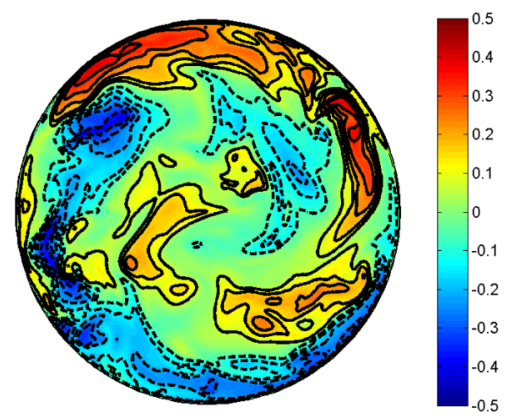

(c) turbulent flow. The precessional force resonates directly with the inertial mode $\boldsymbol{u}_{112}$ but the boundary-layer influx, together with the precessional force, also drives many nonresonant inertial modes that, after the spin-up time scale $O(1 / \sqrt{E})$, become sufficiently strong to be able to interact nonlinearly with the resonant mode $\boldsymbol{u}_{112}$ and lead to strongly turbulent flow. It should be emphasized that a large number of the nonresonant inertial modes having comparable amplitudes, as shown in Table III, are excited. For the numerical experiment with Po $=0.04$ and $E=10^{-4}$, for example, the precessing flow reaches nonlinear equilibrium in the state of strongly turbulent flow when $t>2 / \sqrt{E}$, whose highly irregular structure is depicted in Fig. 8 for four instants.

We now look at the strongly precessing cylinder in the parameter range $(\mathrm{Po} / \sqrt{E})>10$ at $E=10^{-4}$. A surprising and important finding is that the nonlinear flow in the strongly precessing cylinder with $\Gamma=\Gamma_{2}$ becomes weakly turbulent and is marked by its coherent and quasisteady spatial structure at nonlinear equilibrium. When $(\mathrm{Po} / \sqrt{E})>10$, the kinetic energy densities $E_{\text {kin }}$ of the precessing flow, as displayed in Fig. 9 as a function of time for $\mathrm{Po}=0.25$ and $\mathrm{Po}=0.5$, still changes nonperiodically, but with a lower relative amplitude of variation. Its spatial structure, in contrast to that of the strongly turbulent flow shown in Fig. 5, can always be decomposed into two major quasisteady components. First, a weak nonaxisymmetric component largely described by the azimuthal wave number $m=1$ travels retrogradely and is mainly sidewall localized; it is displayed in Fig. 10 at four instants. Apart from time-dependent variation in the form of an azimuthally traveling wave, its main spatial characteristics remain largely unchanged at nonlinear equilibrium. Second, a dominant geostrophic component $U_{G}(s, t)$ in the form of

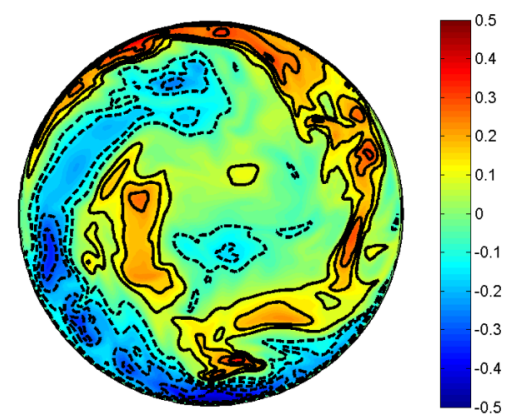

(b)

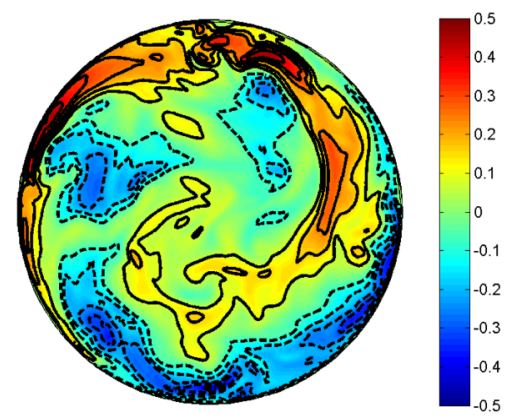

(d)

FIG. 10. (Color online) Contours of $u_{z}$ of the weakly turbulent flow in the mantle frame on the $z=1 / 2$ plane for $\alpha_{p}=\pi / 4$, Po $=0.25$, and $E=10^{-4}$ in the precessing cylinder with $\Gamma=\Gamma_{2}$. 


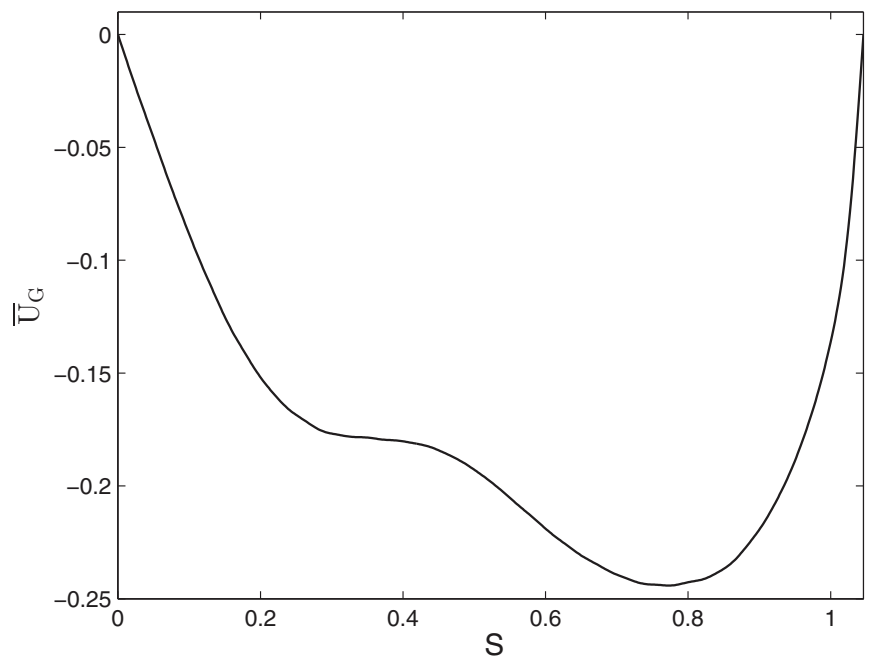

FIG. 11. Averaged axisymmetric geostrophic flow, $\bar{U}_{G}(s)$, as a function of $s$ for $\mathrm{Po}=0.25$ and $E=10^{-4}$ in the precessing cylinder with $\Gamma=\Gamma_{2}$.

differential rotation (not rigid-body rotation) is quasistationary. Figure 11 illustrates its average profile, $\bar{U}_{G}(s)$, defined as

$$
\bar{U}_{G}(s)=\frac{1}{t_{1}-t_{0}} \int_{t_{0}}^{t_{1}} U_{G}(s, t) \mathrm{d} t,
$$

where $\left(t_{1}-t_{0}\right)$ is taken to be about four revolutions. But the average profile $\bar{U}_{G}(s)$ does not significantly depend on the size of $\left(t_{1}-t_{0}\right)$. In comparison to the strongly turbulent precessing flow, the amplitude of the geostrophic flow is much higher than that of the nonaxisymmetric component. At the instant shown in Fig. 10(a), for instance, the resonant mode $\boldsymbol{u}_{112}$ contains he kinetic energy density $E_{112}=0.000416$, while the geostrophic flow $U_{G}$ has $E_{\text {geo }}=0.0021$. Through

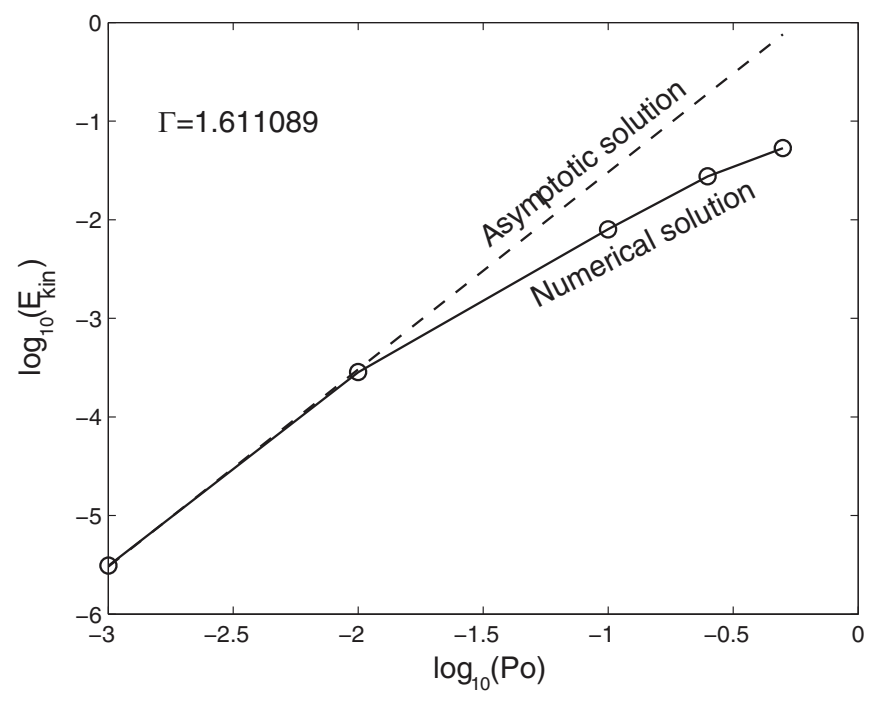

FIG. 12. Kinetic energy densities $E_{\text {kin }}$ plotted as a function of Po for $\alpha_{p}=\pi / 4$ and $E=10^{-4}$ at the third resonance in the precessing cylinder with aspect ratio $\Gamma=\Gamma_{3}$. The dashed line represents the asymptotic solution, while circles denote the results of direct nonlinear numerical simulation.

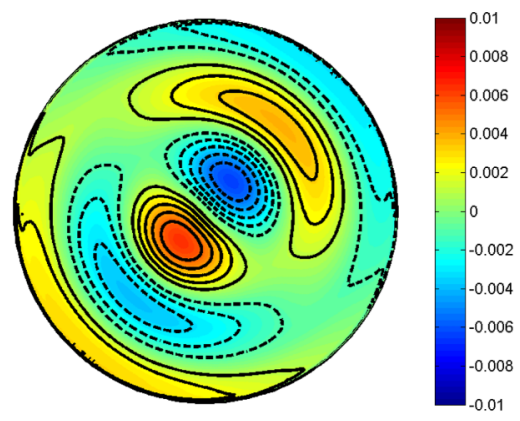

(a)

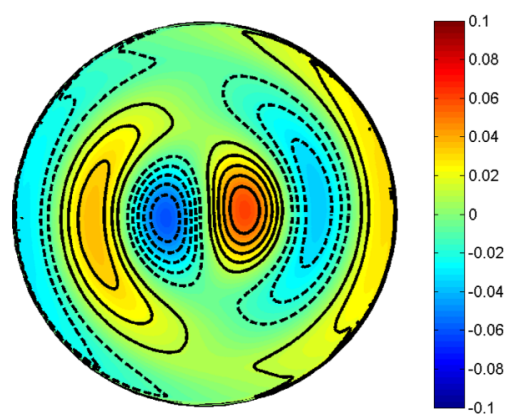

(b)

FIG. 13. (Color online) Contours of $u_{z}$ of the laminar precessing flow with a constant amplitude computed from direct nonlinear numerical simulation in the mantle frame on the $z=1 / 2$ plane for $E=10^{-4}$ in the precessing cylinder at the third resonance with aspect ratio $\Gamma=\Gamma_{3}$ : (a) for $\mathrm{Po}=10^{-3}$ and (b) for $\mathrm{Po}=10^{-2}$.

nonlinear effects in the viscous boundary layers, the energy contained in the resonant mode $\boldsymbol{u}_{112}$ is primarily transferred to the geostrophic flow $U_{G}$. In consequence, the weakly turbulent flow at the leading-order proximation comprises a weak, wall-localized, non-axisymmetric traveling wave mainly with the azimuthal wave number $m=1$ and a strong, quasisteady geostrophic flow $U_{G}$.

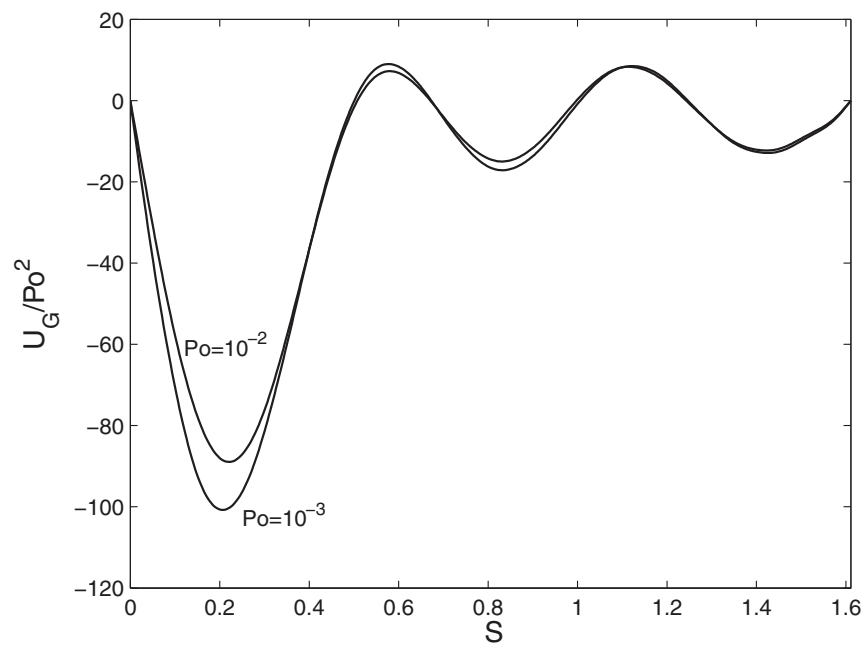

FIG. 14. Scaled axisymmetric, steady geostrophic flow, $U_{G}(s) /$ $\mathrm{Po}^{2}$, as a function of $s$ for $\mathrm{Po}=10^{-3}$ and $10^{-2}$ with $\alpha_{p}=\pi / 4$ and $E=10^{-4}$ at the third resonance in the precessing cylinder with aspect ratio $\Gamma=\Gamma_{3}$. 


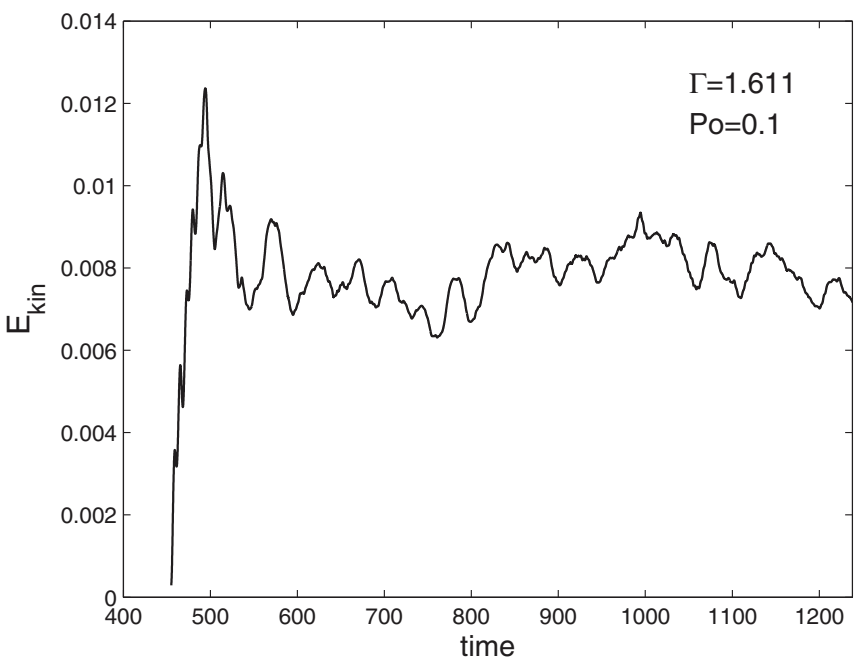

FIG. 15. Kinetic energy densities $E_{\text {kin }}$ of the strongly turbulent precessing flow as a function of time for Po $=0.1$ and $E=10^{-4}$ in the resonant cylinder with aspect ratio $\Gamma=\Gamma_{3}$.

In summary, there exist three regimes in a rapidly rotating cylinder at second resonance with $\Gamma=\Gamma_{2}$. In a weakly precessing cylinder when $0<\mathrm{Po} / \sqrt{E} \leqslant 1$, the precessing flow is laminar with a constant amplitude and dominated by a single resonant inertial mode $\boldsymbol{u}_{112}$ with weak nonresonant inertial modes. When $1<(\mathrm{Po} / \sqrt{E}) \leqslant 10$, while the precessional force still resonates directly with the inertial mode $\boldsymbol{u}_{112}$, many nonresonant inertial modes excited by the precessional force and the boundary-layer influx become strong enough to interact with $\boldsymbol{u}_{112}$, leading to strong turbulence whose randomness and irregularity can only be described in a statistical way. For strongly precessing flow when $(\mathrm{Po} / \sqrt{E})>10$, viscous and nonlinear effects within the viscous boundary layer generate a sufficiently strong geostrophic flow $U_{G}$ that is able to control and stabilize the dynamics of the nonlinear precessing flow, leading to weak turbulence whose leading-order structure is largely regular and quasisteady.

\section{Nonlinear properties at the third resonance}

An extensive computation is also carried out in the precessing cylinder at the third resonance with radius-height aspect ratio $\Gamma=\Gamma_{3}$ whose behavior largely resembles that of the second resonance at $\Gamma=\Gamma_{2}$, and hence, shall keep its discussion brief. In the parameter range $0<\mathrm{Po} / \sqrt{E} \leqslant 1$, the precessing flow is laminar and dominated by a single inertial mode $\boldsymbol{u}_{113}$. As shown in Fig. 12 for $\Gamma=\Gamma_{3}$ at $E=10^{-4}$, there is a good agreement between the asymptotic solution (dashed line) and the nonlinear numerical solutions (represented by circles) for the laminar flow when $0<\mathrm{Po} / \sqrt{E} \leqslant 1$. Displayed in Fig. 13 is the structure of the weakly precessing flow at $\mathrm{Po}=10^{-3}$ and $\mathrm{Po}=10^{-2}$ in a precessing cylinder with $\Gamma=\Gamma_{3}$ obtained from direct nonlinear numerical simulation. Reflecting the more complicated radial structure of the resonant mode $\boldsymbol{u}_{113}$, the axisymmetric, steady geostrophic flow $U_{G}(s)$ for $\Gamma=\Gamma_{3}$ is in the form of differential rotation in alternating eastward and westward directions, which is illustrated in Fig. 14 for $\mathrm{Po}=10^{-3}$ and $\mathrm{Po}=10^{-2}$

In the parameter range $1<\mathrm{Po} / \sqrt{E} \leqslant 10$, many nonresonant inertial modes are excited through the influx from the viscous boundary layers, and consequently, their interaction with the resonant mode leads to strongly turbulent precessing
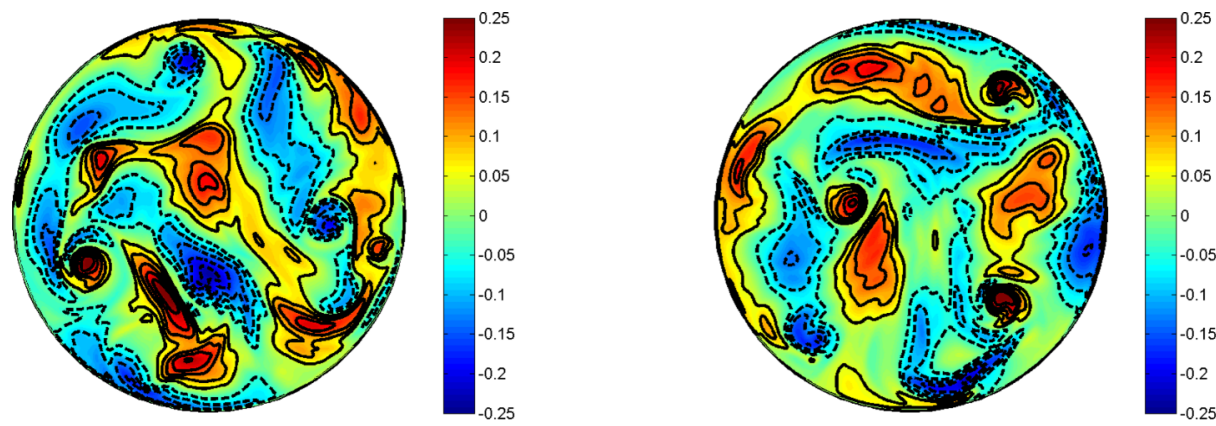

(a)
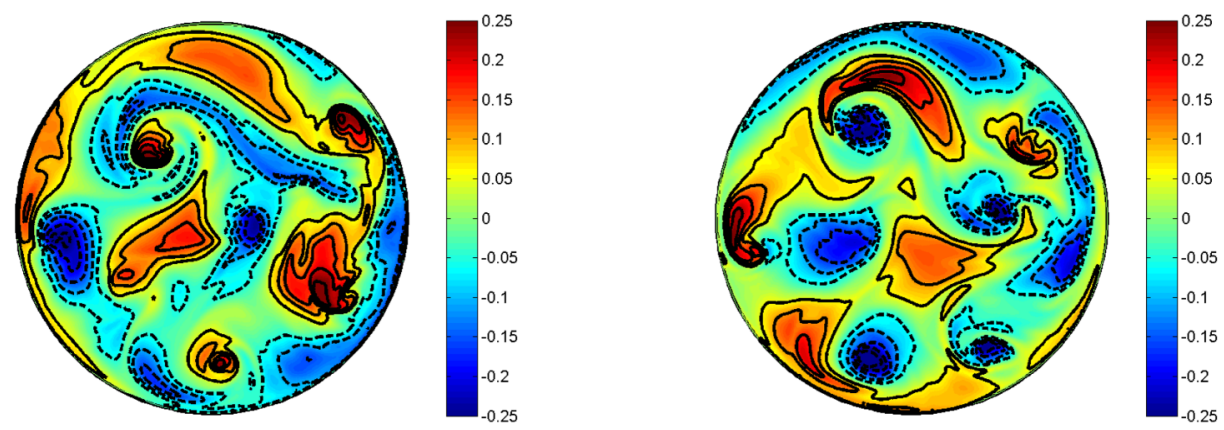

(c)

FIG. 16. (Color online) Contours of $u_{z}$ of the strongly turbulent precessing flow in the mantle frame on the $z=1 / 2$ plane for $\alpha_{p}=$ $\pi / 4, \mathrm{Po}=0.1$, and $E=10^{-4}$ in the precessing cylinder with $\Gamma=\Gamma_{3}$. 
TABLE IV. Largest five coefficients $\left|A_{m n k}\right|$ of the strongly turbulent flow with in the precessing cylinder with $\Gamma=\Gamma_{3}$ and Po $=0.1$ for $E=10^{-4}$ at a typical instant after reaching the nonlinear equilibrium state.

\begin{tabular}{lcc}
\hline \hline$(m, n, k)$ & $\left|\mathcal{A}_{m n k}\right|$ & $\sigma_{m n k}$ \\
\hline$(1,1,3)$ & $1.4863 \times 10^{-1}$ & +0.5000 \\
$(1,0,1)$ & $1.3678 \times 10^{-1}$ & +0.0000 \\
$(1,1,1)$ & $1.3419 \times 10^{-1}$ & +0.9001 \\
$(1,1,2)$ & $1.1864 \times 10^{-1}$ & +0.6701 \\
$(4,0,1)$ & $8.8406 \times 10^{-2}$ & +0.0000 \\
\hline \hline
\end{tabular}

flow. As an example of strong turbulence, the kinetic energy densities $E_{\mathrm{kin}}(t)$ and the profiles of the flow at several instants are presented in Figs. 15 and 16 at $\mathrm{Po}=0.1$ and $E=10^{-4}$ with $\Gamma=\Gamma_{3}$. When $t=O(1 / \sqrt{E})$, the nonresonant inertial modes become sufficiently strong to interact nonlinearly with the resonant mode $\boldsymbol{u}_{113}$. A typical spectrum of the five largest coefficients $\left|A_{m n k}\right|$ is presented in Table IV when the system attains nonlinear equilibrium. It should be noted that the geostrophic flow $\mathcal{A}_{G}$ is too weak to be listed in the table: $\mathcal{A}_{G}=0.006$, while $\mathcal{A}_{113}=0.1486$. Again, there do not exist two free inertial modes at any instants that are sufficiently strong to form triadic resonance with the resonant mode $\boldsymbol{u}_{113}$. While the precessional force resonates directly with the inertial mode $\boldsymbol{u}_{113}$, many nonresonant inertial modes also become sufficiently strong. It is the nonlinear interactions between the resonant mode $\boldsymbol{u}_{113}$ and many nonresonant inertial modes, such as $\boldsymbol{u}_{111} \boldsymbol{u}_{112}$, that result in the strongly turbulent flow depicted in Fig. 16.

When the cylinder is precessing strongly with $(\mathrm{Po} / \sqrt{E})>$ 10 , the strongly precessing flow becomes weakly turbulent. The kinetic energy densities $E_{\text {kin }}$ for Po $=0.25$ and $\mathrm{Po}=0.5$ with $\Gamma=\Gamma_{3}$ are displayed in Fig. 17 as a function of time. Its corresponding spatial structure after reaching nonlinear equilibrium comprises two major components at the leadingorder approximation: a dominant geostrophic component $U_{G}$,

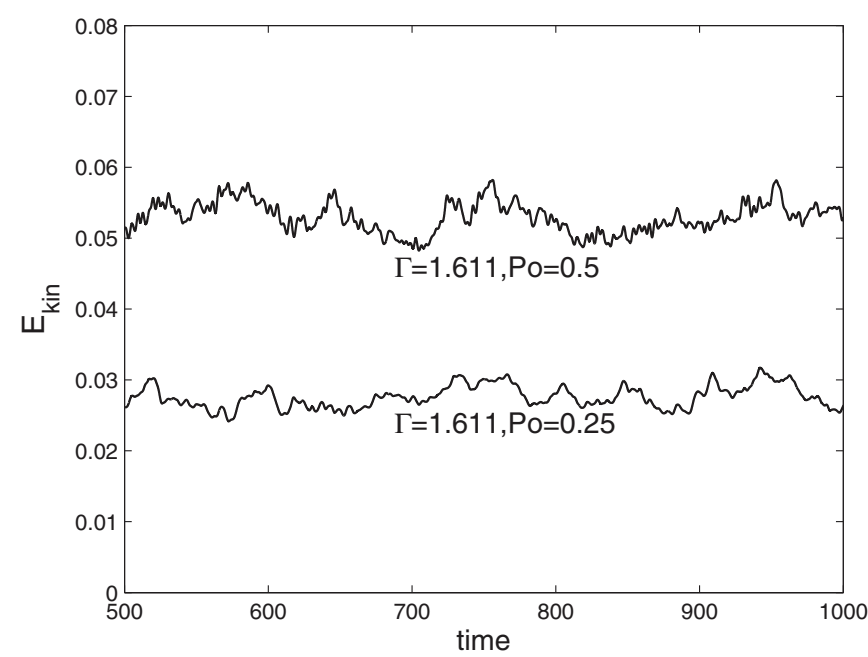

FIG. 17. Kinetic energy densities $E_{\text {kin }}$ of the weakly turbulent precessing flow as a function of time for $\mathrm{Po}=0.25$ and $\mathrm{Po}=0.5$ at $E=10^{-4}$ in the third resonant cylinder with $\Gamma=\Gamma_{3}$.

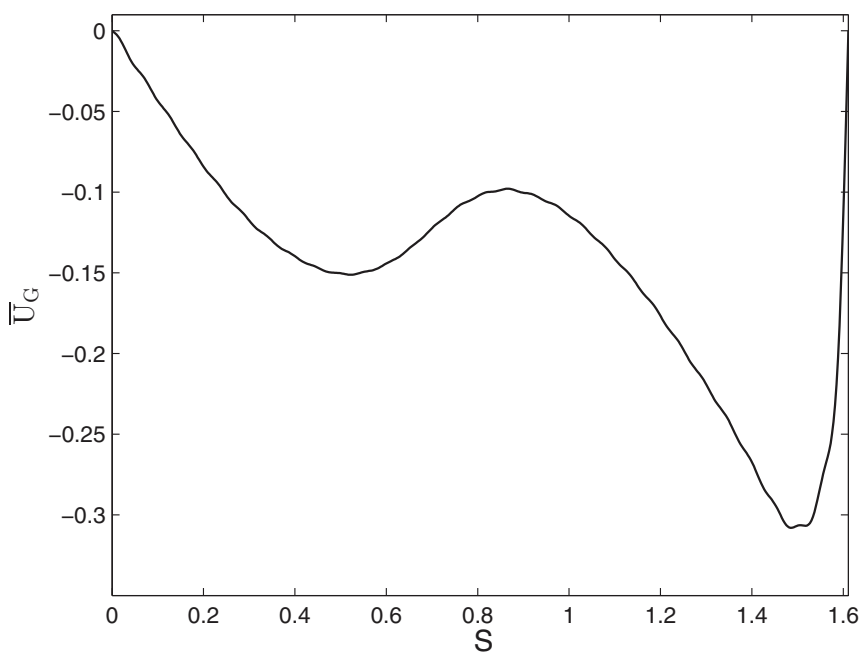

FIG. 18. Averaged geostrophic flow, $\bar{U}_{G}(s)$, as a function of $s$ for Po $=0.5$ for $\alpha_{p}=\pi / 4$ and $E=10^{-4}$ in a precessing cylinder with aspect ratio $\Gamma=\Gamma_{3}$.

in the form of differential rotation, whose average profile $\bar{U}_{G}(s)$ is depicted in Fig. 18; and a weak nonaxisymmetric component chiefly described by the azimuthal wave number $m=1$ and largely localized in the vicinity of the sidewall where the geostrophic flow $U_{G}$ is weak. A typical profile of the weakly turbulent flow for Po $=0.5$ is displayed in Fig. 19 at two instantsat nonlinear equilibrium; its primary features remain nearly unchanged in the drifting frame of a retrogradely

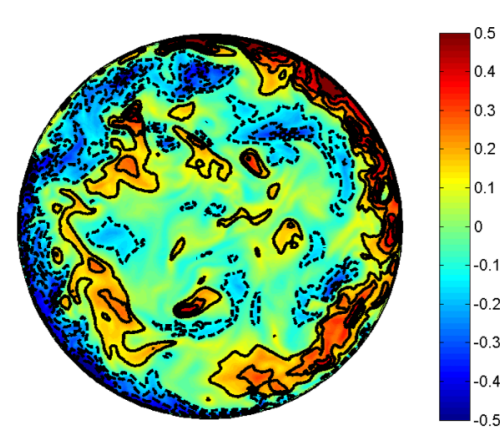

(a)

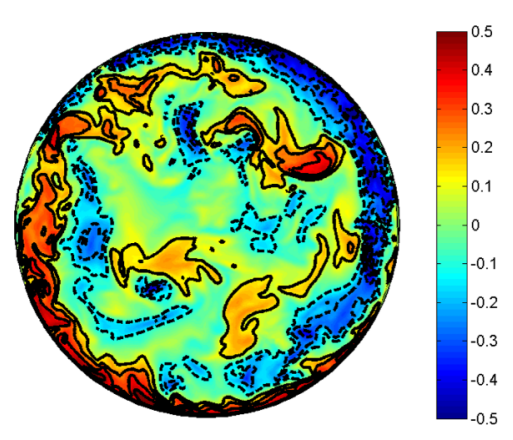

(b)

FIG. 19. (Color online) Contours of $u_{z}$ of the weakly turbulent precessing flow in the mantle frame on the $z=1 / 2$ plane for $\alpha_{p}=\pi / 4$, Po $=0.5$, and $E=10^{-4}$ in the precessing cylinder with $\Gamma=\Gamma_{3}$. 
traveling wave. At the instant shown in Fig. 19(a), the geostrophic flow $U_{G}$ contains the kinetic energy $E_{\text {geo }}=0.020$, while the resonant mode $\boldsymbol{u}_{113}$ has only $E_{113}=0.0033$. As the geostrophic flow $U_{G}$ becomes sufficiently strong to be capable of controlling its nonlinear dynamics, the strongly precessing flow becomes weakly turbulent.

\section{SUMMARY AND REMARKS}

Our investigation, via both asymptotic analysis and direct numerical simulation, has focused on precessionally driven flow in two cylinders marked by radius-height aspect ratios $\Gamma=\Gamma_{2}$ and $\Gamma=\Gamma_{3}$ at which Poincaré forcing resonates directly with inertial modes $\boldsymbol{u}_{112}$ and $\boldsymbol{u}_{113}$, respectively. Three nonlinear regimes for Po are identified at an asymptotically small Ekman number, $0<E \ll 1$. First, when the cylinders are precessing weakly with $0<(\mathrm{Po} / \sqrt{E})<1$, the precessing flow, dominated by a single resonant inertial mode, $\boldsymbol{u}_{112}$ or $\boldsymbol{u}_{113}$, is laminar with a constant amplitude and the asymptotic analytical solution shows a quantitative agreement with the nonlinear numerical solution of direct simulation. Second, in the parameter range $1<\mathrm{Po} / \sqrt{E} \leqslant 10$ at a moderate rate of precession, a large number of the nonresonant modes with comparable amplitudes, in addition to the resonant inertial mode, are excited via viscous and nonlinear effects, but the geostrophic flow $U_{G}$ remains weak. There is a net energy cascade from the precession-driven inertial wave at the near-domain length scale to smaller scales due to quadratic nonlinearity in the governing equation. Third, when the cylinder is precessing strongly in the approximate range $(\mathrm{Po} / \sqrt{E})>10$, the geostrophic flow $U_{G}$, which can be readily generated and maintained by nonlinear effects in the viscous boundary layers at the top and bottom of the cylinder as Po increases, becomes strong and predominant. Suppose that the precessing flow to leading-order approximation is expressible as

$$
\boldsymbol{u}=U_{G} \hat{\boldsymbol{\phi}}+\widehat{\boldsymbol{v}},
$$

where $\widehat{\boldsymbol{v}}$ denotes the weak nongeostrophic component with

$$
\left|U_{G}\right| \gg|\widehat{\boldsymbol{v}}| \text {. }
$$

It follows that the nonlinear term in the governing equation, (1), can be written as

$$
\boldsymbol{u} \cdot \nabla \boldsymbol{u}=\left(U_{G} \hat{\boldsymbol{\phi}}+\widehat{\boldsymbol{v}}\right) \cdot \nabla\left(U_{G} \hat{\boldsymbol{\phi}}+\widehat{\boldsymbol{v}}\right) \approx\left(\frac{U_{G}^{2}}{s}\right) \widehat{\boldsymbol{s}},
$$

which can be largely balanced by the pressure gradient. Since the ageostrophic component $\widehat{v}$ of the precessing flow remains saturated as Po increases, it is the geostrophic flow $U_{G}$ that controls the nonlinear dynamics in the region of the cylinder where $U_{G}$ is predominant.
The nonlinear results presented in this paper are fairly robust even though we have concentrated on the second and third resonances with radius-height aspect ratios $\Gamma=$ $\Gamma_{2}=1.045945$ and $\Gamma=\Gamma_{3}=1.611089$ at a high precision. This is because the general property of the nonlinear flow obtained at $\Gamma=1.045945$ or $\Gamma=1.611089$ would be largely similar to that calculated at slightly different aspects, for example, $\Gamma=1.0$ or $\Gamma=1.6$. In other words, the main conclusions for the nonlinear (strongly precessing) problem do not require the high precision of the radius-height aspect ratio $\Gamma$. Mathematically speaking, however, a highly accurate $\Gamma$ is required for the linear (weakly processing) problem in the asymptotic expansion, (11), that is valid only at resonance for $0<E \ll 1$ and $0<(\mathrm{Po} / \sqrt{E}) \ll 1$.

To capture the effect of an active viscous boundary layer at resonance, direct numerical simulation must run longer than the spin-up time $O\left(E^{-1 / 2}\right)$ so as to reach a nonlinear equilibrium state. This results in prohibitively high computational costs for very small values of the Ekman number. Since the asymptotic analytical solution valid only for $0<E \ll 1$ shows a quantitative agreement with the numerical solutions at $E=10^{-4}$, in this paper we have thus concentrated on the numerically less expensive case with $E=10^{-4}$. However, we did perform several numerical simulations at $E=5 \times 10^{-5}$ in the precessing cylinder with $\Gamma=\Gamma_{2}$, confirming that the nonlinear behavior at $E=5 \times 10^{-5}$ is largely the same as that obtained at $E=10^{-4}$.

The finding that the geostrophic flow $U_{G}$ is capable of controlling/stabilizing the nonlinear dynamics of precessing flow in the resonant cylinders and, hence, determines the nature of turbulence may have an implication for the understanding of the general flow character in rapidly rotating fluid systems. Many rapidly rotating planets such as Jupiter and Saturn share the same essential feature: they have a strong geostrophic flow (zonal winds) that is stable and in a weakly turbulent state (in the sense that its leading-order structure is regular and quasisteady). Our results seem to support the conjecture that, if a rapidly rotating fluid system has a mechanism that can effectively generate and maintain a strong geostrophic flow, it will be unable to attain a strongly turbulent state at its nonlinear equilibrium.

\section{ACKNOWLEDGMENTS}

K.Z. was supported by Leverhulme Trust Research Project Grant No. RPG-2015-096 and by Macau FDCT Grant No. 039/2013/A2. R.Z. was supported by NSFC Grant No. 41274073. The numerical simulation was supported by the high-performance computing facilities at Institute of Geology and Geophysics, Chinese Academy of Sciences.
[1] W. W. Wood, An oscillatory disturbance of rigidly rotating fluid, Proc. R. Soc. Lond. A 293, 181 (1966).

[2] R. F. Gans, On the precession of a resonant cylinder, J. Fluid Mech. 41, 865 (1970).
[3] R. Manasseh, Breakdown regimes of inertia waves in a precessing cylinder, J. Fluid Mech. 243, 261 (1992).

[4] J. J. Kobine, Inertial wave dynamics in a rotating and precessing cylinder, J. Fluid Mech. 303, 233 (1995). 
[5] P. Meunier, C. Eloy, R. Lagrange, and F. Nadal, A rotating fluid cylinder subject to weak precession, J. Fluid Mech. 599, 405 (2008).

[6] T. Lehner, W. Mouhali, J. Leorat, and A. Mahalov, Mode coupling analysis and differential rotation in a flow driven by a precessing cylindrical container, Geophys. Astrophys. Fluid Dynam. 104, 369 (2010).

[7] X. Liao and K. Zhang, On flow in weakly precessing cylinders: the general asymptotic solution, J. Fluid Mech. 709, 610 (2012).

[8] W. Mouhali, T. Lehner, J. Leorat, and R. Vitry, Evidence for a cyclonic regime in a precessing cylindrical container, Exp. Fluids 53, 1693 (2012).

[9] Y. Lin, J. Noir, and A. Jackson, Experimental study of fluid flows in a precessing cylindrical annulus, Phys. Fluids 26, 046604 (2014).

[10] M. Pozzo, C. Davies, D. Gubbins, and D. Alfè, Thermal and electrical conductivity of iron at Earth's core conditions, Nature 485, 355 (2012).

[11] W. V. R. Malkus, Precession of the earth as the cause of geomagnetism, Science 160, 259 (1968).

[12] C. A. Dwyer, D. J. Stevenson, and F. Nimmo, A long-lived lunar dynamo driven by continuous mechanical stirring, Nature 479, 212 (2011).

[13] C. Nore, J. Léorat, J.-L. Guermond, and F. Luddens, Nonlinear dynamo action in a precessing cylindrical container, Phys. Rev. E 84, 016317 (2011).

[14] A. Tilgner, Precession driven dynamos, Phys. Fluids 17, 034104 (2005).

[15] C. C. Wu, and P. H. Roberts, On a dynamo driven by topographic precession, Geophys. Astrophys. Fluid Dynam. 103, 467 (2009).
[16] J. Boisson, D. C. Cébron, F. Moisy, and P.-P. Cortet, Earth rotation prevents exact solid-body rotation of fluids in the laboratory, Europhys. Lett. 98, 59002 (2012).

[17] S. A. Triana, D. S. Zimmerman, and D. P. Lathrop, Precessional states in a laboratory model of the Earth's core, J. Geophys. Res. 117, B04103 (2012).

[18] Lord Kelvin, Vibrations of a columnar vortex, Philos. Mag. 10, 155 (1880).

[19] H. P. Greenspan, The Theory of Rotating Fluids (Cambridge University Press, Cambridge, UK, 1968).

[20] K. Zhang and X. Liao, On the initial value problem in a rotating circular cylinder, J. Fluid Mech. 610, 425 (2008).

[21] K. Zhang, K. Chan, and X. Liao, On precessing flow in an oblate spheroid of arbitrary eccentricity, J. Fluid Mech. 743, 358 (2014).

[22] D. Kong, X. Liao, and K. Zhang, The sidewall-localized mode in a resonant precessing cylinder, Phys. Fluids 26, 051703 (2014).

[23] D. Kong, Z. Cui, X. Liao, and K. Zhang, On the transition from the laminar to disordered flow in a precessing spherical-like cylinder, Geophys. Astrophys. Fluid Dynam. 109, 62 (2014).

[24] K. H. Chan, K. Zhang, and X. Liao, An EBE finite element method for simulating nonlinear flows in rotating spheroidal cavities, Int. J. Numeric. Methods Fluids 63, 395 (2010).

[25] K. H. Chan, Y. He, K. Zhang, and J. Zou, A finite element analysis on fluid motion in librating triaxial ellipsoids, Numeric. Methods Part. Dif. Eq. 30, 1518153 (2014).

[26] Z. Cui, K. Zhang, and X. Liao, On the completeness of inertial wave modes in rotating annular channels, Geophys. Astrophys. Fluid Dynam. 108, 44 (2014). 